\title{
Interval Type-2 Fuzzy Sets Improved by Simulated Annealing for Locating the Electric Charging Stations
}

\author{
Seda Türk ${ }^{\mathrm{a}}$, Muhammet Deveci*b,c ${ }^{*}$ Ender Özcan $^{\mathrm{c}}$, Fatih Canıtez $^{\mathrm{d}}$, Robert \\ $\mathrm{John}^{\mathrm{c}}$ \\ ${ }^{a}$ Department of Industrial Engineering, Faculty of Engineering, Igdir University, 76000 \\ Merkez, Igdir, Turkey \\ ${ }^{b}$ Department of Industrial Engineering, Naval Academy, National Defense University, \\ 34940 Tuzla, Istanbul, Turkey. \\ ${ }^{c}$ Computational Optimisation and Learning (COL) Lab, School of Computer Science, \\ University of Nottingham, NG8 1BB, Nottingham, UK. \\ ${ }^{d}$ Department of Management Engineering, Faculty of Management, University of Istanbul \\ Technical, 34367 Maka, Istanbul, Turkey
}

\begin{abstract}
Electric vehicles are the key to facilitating the transition to low-carbon 'green' transport. However, there are concerns with their range and the location of the charging stations which delay a full-fledged adoption of their use. Hence, the electric charging infrastructure in a given region is critical to mitigating those concerns. In this study, an interval type-2 fuzzy set based multi-criteria decision-making method is introduced for selecting the best location for electric charging stations. This method is improved by Simulated Annealing obtaining the best configuration of the parameters of the interval type- 2 membership functions along with two different aggregation operators; linguistic weighted sum and average. The proposed overall reusable multi-stage solution approach is applied to a real-world public transport problem of the municipal bus company in Istanbul. The results indicate that the approach indeed improves the model, capturing the associated uncertainties embedded in the interval type-2 membership functions better, leading to a more effective fuzzy system. The
\end{abstract}

\footnotetext{
* Corresponding author

Email addresses: sedaturk@hotmail.com (Seda Türk),

muhammetdeveci@gmal.com;muhammet.deveci@nottingham.ac.uk (Muhammet Deveci*), ender.ozcan@nottingham.ac.uk (Ender Özcan), fatihcanitez40@gmail.com (Fatih Canitez), robert.john@nottingham.ac.uk (Robert John)
}

Preprint submitted to Information Sciences 
experts confirm those observations and that Simulated Annealing improved interval type-2 fuzzy method achieves more reliable results for selecting the best sites for the electric bus charging stations.

Keywords: Facility location, site selection, interval type-2 fuzzy sets, multi-criteria decision-making, simulated annealing.

\section{Introduction}

Electric vehicles are gaining more and more attention from the governments and general public across the world. This is mainly due to the growing sustainability concerns. With the improvements in the battery technology, electric vehicles have become a promising solution to addressing some of the vital environmental challenges, such as fossil resource depletion 11. Many public bus operators have already started to replace the conventional buses running on fossil fuels with electric buses to encourage environmentally friendly transport modes contributing to the sustainable urban development. However, that transition to electric buses requires making some infrastructural investments, such as, building electric charging stations, and determining the optimal locations for those stations [2].

Despite the decrease in the battery costs in recent years, the battery is still one of the most expensive and restricting components of electric vehicles. Many electric vehicles have a limited driving range with full charge when compared to the internal combustion engine-powered vehicles with full tank [3]. Recharging a battery is a time-consuming activity in electric bus operations. There are alternative solutions to this issue, such as battery swapping [4] and dynamic wireless recharging [5]. However, there is still the issue of where to deploy electric charging stations, especially when there is an existing infrastructure as in the case of public transport.In this study, we address the issue of determining the optimal location for electric charging stations as a decision-making problem for the public bus operators. 


\subsection{Approaches to Location Selection Problems}

There are various previous studies tackling the problem of optimal location selection for the electric charging stations, and applying exact and inexact solution methods, including the mixed-integer nonlinear programming and Bendersand-Price algorithm [3], Trip Success Ratio approach [2], bi-level programming model approach [6], genetic algorithm [7], and more. Although type-2 fuzzy sets have been applied to a range of location selection problems from ambulance location selection [8] to underground storage site selection [9], there are a few studies relevant to the electric charging stations.

Multi-Criteria Decision-Making (MCDM) methods have been proposed for different electric charging location selection problems, many of which focus mainly on certain regions in China. Guo and Zhao [1] applied a fuzzy TOPSIS method for site selection with 3 main criteria and 11 sub-criteria as decision factors to choose one of the 4 alternative locations in Beijing, China. Wu et al. [10] utilized a Preference Ranking Organization Method for Enrichment Evaluations combined with the cloud model and Analytic Network Process in Beijing, China. Zhao and Li [7] employed fuzzy Grey relation analysis VIseKriterijumska Optimizacija I Kompromisno Resenje (VIKOR) method to determine the optimal electric charging station location in Tianjin, China. Xu et al. 11] used an interval type-2 fuzzy method to determine the optimal location for electric charging stations for a state company in Tianfu New District, China. They ensured the rationality of their findings through the entropy weight method - a rough consensus reaching process. Interval type-2 fuzzy is applied to model the human language for the EVCS site selection problem to capture the vagueness and uncertainty in decision-makers' preferences. Wu et al. [12 proposed a Fuzzy VIKOR with 5 main criteria and 16 sub-criteria for 6 alternative locations in Beijing, China. Cui et al [13] proposed a Pythagorean fuzzy VIKOR method to site selection problem for electric vehicle charging stations with a practical example in Shanghai, China. Interval type-2 fuzzy ELECTRE method was applied to the allocation problem of the electric charging station [14. Karasan et al. [15] used an integrated MCDM methodology with intuitionistic fuzzy sets on 
the electric charging station selection problem.

To the best of authors' knowledge, this is one of the first studies applying interval type-2 fuzzy sets improved by a local search metaheuristic to the electric charging station selection problem. The proposed approach is employed to solve a particular public transport real-world problem instance from Turkey.

\subsection{The motivation for using Interval Type-2 Fuzzy Sets}

Due to the uncertain nature of the decision making process based on various criteria and their variability for electric charging location selection, fuzzy sets have been used as a crucial part of the solution methods. Zadeh [16] proposed type-2 fuzzy sets that have additional degrees of freedom to model the problems in which type-1 fuzzy sets are not capable of handling adequately.

The scientific literature shows that type-2 fuzzy sets have more advantages over the type-1 fuzzy sets. Firstly, numerical and linguistic uncertainties can be handled effectively, since the membership functions themselves are defined as fuzzy sets. Secondly, wider coverage of uncertainties is provided by type-2 fuzzy sets with fuzzy membership functions while reducing the number of rules used [17]. Finally, the extra dimension is likely to enhance the performance of a type-2 fuzzy logic system while a type-1 fuzzy logic system cannot provide the same result under the same conditions [18].

Nevertheless, type-reduction and defuzzification processes in the applications of type-2 fuzzy logic systems often require additional time. Because of the computational complexity of those processes, the majority of the researchers and practitioners have focused on interval type-2 fuzzy models [19] with manageable run-time complexities that are considered practical [20, 21]. [20] defines the foot print of uncertainty (FOU) as "the union of the primary membership". In general, there is no restriction on the shape of FOUs for the type-2 sets. This could lead to the loss of the semantic relationship between the type- 2 fuzzy set and the concept that it models [22. However, the values of secondary memberships are restricted to either zero or one in the interval type-2 fuzzy sets, enabling parameterization of the primary membership functions (and so FOUs), 
and resolution of the semantic issues [23. Larger FOUs in the applications of interval type-2 fuzzy sets are not preferable as they represent higher level of uncertainty characterized by the problem [24]. Then again, expanding FOUs to some extent could have a positive effect on capturing the uncertainty [25]. Therefore, in this study, we have used a local search metaheuristic searching for the best primary membership functions with reasonable FOUs for the location selection problem of electric charging stations. Additionally, Dalterio et al. [22] highlighted that the semantic relationship can be lost easily in a fuzzy rule-based system. Hence, the proposed overall method using interval type-2 trapezoidal fuzzy sets applies computing with words approach investigating two aggregation approaches based on linguistic weighted sum and average keeping the semantic relation with the concept.

\subsection{Improving the Performance of Fuzzy Systems}

In this study, simulated annealing (SA) [26] as a local search metaheuristic [27] is presented to optimise and find the best parameter settings for the fuzzy interval type-2 membership functions. There are previous studies that investigate the influence of parameter settings of fuzzy systems on their performance. The majority of that work focuses on parameter tuning, i.e. finding the best initial parameter settings for fuzzy logic controllers as the application area.

In 1993, Metvally and Malik [28] observed that parameter tuning is an essential process and has the potential to improve the performance of fuzzy logic control and so they proposed a technique to cope with system imprecision, system ill-definition, and uncertainty to achieve the desired system response.

Different granular approaches which divide the design of the global controller into several individual simpler controllers are used on the generalized type2 fuzzy systems [29], interval type-2 fuzzy sets [30, type-2 fuzzy logic [31, comparative studies of type-1 fuzzy logic systems, interval type- 2 fuzzy logic systems and generalized type-2 fuzzy logic systems [32, 33. Melin et al. [34] pointed out the uncertainty in fuzzy logic controllers and fuzzy edge detectors and improved to model general type-2 fuzzy logic system based on shadowed 
type-2 fuzzy membership functions for the control problems.

The majority of the previous approaches were applied to control problems where there is a high level of uncertainty. It has been observed that the proposed algorithm improving the logic controllers results in better performance. In this study, we investigate parameters of interval type-2 fuzzy sets rather than the parameters related to the application (e.g., logic controllers).

\subsection{The motivation for using Simulated Annealing}

Finding the best metaheuristic approach for solving a problem itself is a challenging task since this requires even further experimentation with different metaheuristics and parameter tuning of those metaheuristics. So, we referred to the scientific literature. The previous work [35, 36] shows that SA is an easy-toimplement approach with fewer parameters to tune as compared to some other metaheuristics, but more importantly, it is an extremely effective metaheuristic capable of escaping from local optima. [36 tested SA as a general-purpose search method across nine different problem domains and reported the success of SA when compared to a variety of other metaheuristics. Hence, we preferred SA for improving the interval type-2 membership functions. Then the

electrical charging station selection problem is solved using the best parametric configuration obtained from SA.

\subsection{Objectives of the Study}

The majority of the previous work on location selection for the charging stations involving the use of MCDM methods are applied to specific problems from China. Moreover, they all address the relevant problems for the electric "vehicles" not "buses" for public transport, which differ based on a different set of criteria and geographical constraints. In this study, we introduce a novel multi-stage approach based on interval type-2 fuzzy sets improved by SA for determining the best location for the electric charging stations for electric buses, which will be purchased by IETT, the municipal public transport operator of Istanbul in Turkey. The main objectives of this study are to: 
- find the best location for the electric charging stations for electric buses using interval type-2 fuzzy sets,

- investigate uncertainty in 'computing with words' using two different aggregation approaches namely linguistic weighted sum and average, and

- find out the best configuration for the fuzzy sets for the importance of criteria using SA to cope with the ambiguity on subjective judgements.

The problem of finding the best location for the electric charging stations for electric buses is formulated as a multi-criteria decision-making problem considering that there are many decision factors/criteria involved. Uncertainty plays an important role in this decision-making problem since criteria are far from being certain and involve decision makers subjective judgments. Hence, we have tested interval type-2 fuzzy sets to evaluate alternative garages based on two different aggregation operators; linguistic weighted sum and average. Besides, the membership functions are improved and configured to handle uncertainty within the fuzzy sets using a well-known metaheuristic - Simulated Annealing (SA). Using the best configuration obtained by SA for the membership functions, the approach based on the interval type- 2 fuzzy sets is applied to the real-world problem, and performances of the two aggregation methods are compared. The overall approach is sufficiently general and reusable, that is, it can be applied to any multi-criteria decision making problem.

The rest of the paper is structured as follows. Section 2 introduces the realworld problem including its description and the decision criteria. In Section 3 the overall approach is described focusing on each algorithmic component. Section 4 discusses the results from the computational experiments, summarising the parameter tuning results for SA and illustrating the performance of SA improved approach based on the interval type-2 fuzzy sets as compared to the approach which does not utilise SA. Finally, Section 6 concludes the study and presents some potential future research directions. 


\section{Problem Description}

Istanbul, with a population reaching 15 million, is the economic, cultural, and tourism capital of Turkey. Istanbul has a well-integrated public transit system, comprising of public buses, metro, light rail, street tramway, ferry, and bus rapid transit (BRT). IETT is the municipal public transport company operating 2,236 public and 510 BRT buses. BRT has 52-kilometers length, extending over one of the most crowded road corridor (D-100) of Istanbul. It has 45 stations and daily serves over 900,000 passengers. Figure 1 shows the outline of BRT line, as completed phase by phase from 2007 to 2012. BRT passenger revenues comprise $56 \%$ of IETT's total revenues. Besides its financial importance, BRT is important to provide an integrated, safe and fast transport option for people and reputationally crucial for IETT.

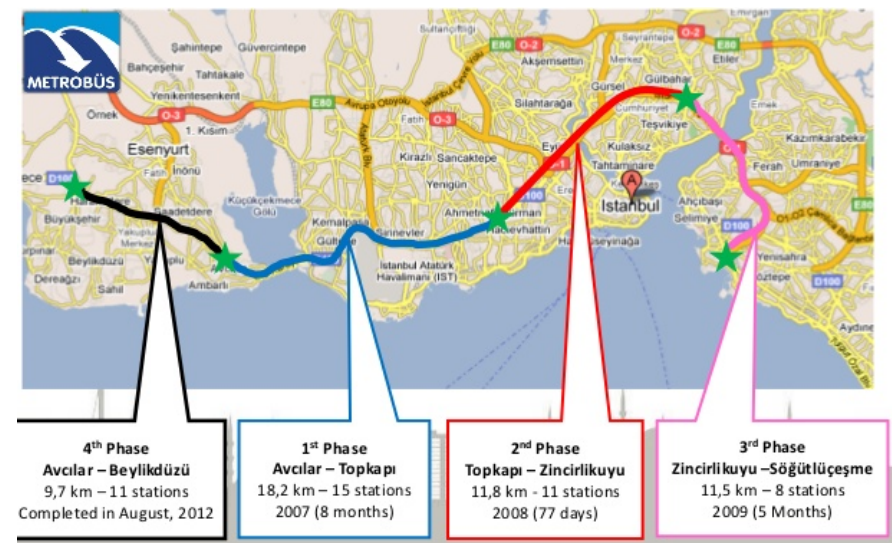

Figure 1: Istanbul bus rapid transit (BRT) 37.

The bus fleet is composed mainly of diesel (79\%) and CNG (21\%) buses. Due to IETT's strategy to become a leader in sustainable bus operations in Turkey, it plans to renew its bus fleet, gradually replacing diesel buses with electric buses. Initially, 10 electric buses are planned to be added to the fleet. Those electric buses will be mainly used in BRT operations, not excluding the possibility of using them in regular bus services. 
Selecting the most appropriate bus garages for placing the electric charging stations for the electric buses involves multiple criteria and it is a challenging task. In the following sections, we present the criteria considered in our approach for tackling this problem along with the bus garages based on real-world data.

\subsection{Criteria for Decision Making}

We identified 5 main criteria grouping 14 qualitative sub-criteria for this decision-making problem, drawn from both extant literature and expert opinion from IETT. Some of the criteria are specifically identified for this problem which are referred to as context-specific criteria: closeness to BRT and Interchange Stations, closeness to bus routes, availability of garage space, maintenance capability, and revenue potential. The distinguishing feature of the problem in this study is that it deals with a public bus operator's future electric bus and charging station investment for its BRT operations. We also identified 5 out of 14 IETT garages for locating electric charging stations. These alternatives will be assessed based on their performance regarding the decision criteria by 4 decision-makers. Those decision-makers are the senior managers of IETT having a decision-making power regarding the electric bus purchase. Their positions are the following: Deputy General Manager responsible for technical issues, Head of Technology Development Department, Head of Maintenance Department, bus operations expert in Fleet Operations Department.

The main criteria and sub-criteria can be briefly summarized as follows:

Economy: Economic evaluations are important in electric charging station investments. The cost and revenue of investments are key determinants of economic performance. Therefore, the investment payback period and revenue potential are considered as the economic decision criteria.

(C1) Investment payback period: This entity refers to the total investment cost divided by annual returns. Returns from electric charging stations can be calculated by savings gained through using electricity rather than petrol or diesel expenses as well as passenger revenues using those electric vehicles. Using the investment pay-off period assesses of gains from electric charging stations 
viable [10].

(C2) Revenue potential (Closeness to dense passenger area): The number of users benefiting from electric buses provides ticket revenues for the bus operator. If an electric charging station is closer to the denser passenger areas where ticket revenues are likely to be more, then the revenue potential would increase.

Energy: Access to energy sources is critical for electric charging stations. Therefore; closeness to a power grid, reliability of electricity power, distance from heavy electricity consumption and availability of renewable energy resources are important to ensure a seamless electricity feed to the charging stations. While selecting a location, those factors would play a crucial role.

(C3) Closeness to power grid: The electric charging station should be sufficiently close to the power grid so to ensure adequate energy input.

(C4) Reliability of electricity power: Electricity coming from the energy grid should be reliable and stable to ensure the secure operation of the distribution network.

(C5) Distance from heavy electricity consumption: A large number of charging users harms power stability and quality [12], therefore, electric charging stations should be away from heavy load-lines.

(C6) Availability of renewable energy resources: To ensure a sustainable energy input, the availability of renewable energy resources, such as solar panels, wind turbines or biofuel is important.

Service Area: The features of the service area where electric charging stations are located influence the effectiveness of the electric charging scheme.

(C7) Closeness to BRT or Interchange Stations: Since electric buses are planned by IETT to be used in BRT services, the closeness of electric charging stations to BRT stations is also important.

(C8) Traffic congestion: Traffic congestion makes electric vehicles consume more electric, therefore, the service area where there is low traffic congestion is preferred [1, 7, 11].

(C9) Closeness to bus routes: Besides BRT service, the electric buses can also be deployed in regular bus services, therefore, it is preferable to have electric 
charging stations to the bus routes to decrease the deadheading kilometer.

(C10) Topographical properties (high slope): Higher slope makes electric vehicles consume more electricity, therefore, the areas where there is the low slope are more appropriate for locating electric charging stations [10].

Garage Capacity: Having adequate garage capacity is important to be able to host electric buses and charging stations. Besides enough garage space, expandability of the garage area in case of future fleet expansions and having sufficient maintenance capability are other factors enabling the introduction of the electric charging stations.

(C11) Availability of garage space: The garage must have enough space to put electric charging stations.

(C12) Expandability: In case of future expansions, the garage must have the flexibility for area expansions.

(C13) Maintenance capability: Electric charging stations require planned and unplanned maintenance, therefore, having a maintenance capability in terms of financial, human, and technical know-how resources is crucial to ensure the functioning of electric charging stations [1, 10].

Safety: Protection of electric charging stations against acts of vandalism, theft, or other attacks is important.

(C14) Security infrastructure: Having a security infrastructure (security staff, CCTV cameras, etc.) is desirable to ensure a safe environment for electric charging stations.

\subsection{Alternative Options}

We have five alternative garages to consider to place the electric charging stations for the electric buses based on the real-world data obtained from IETT as illustrated in Figure 2 2 Table 1 summarises the relevant features of those garages 37. The detailed description of each garage is given below.

- Ikitelli: The largest garage in IETT is Ikitelli garage, located in the western part of Istanbul on a large area $\left(196,322 \mathrm{~m}^{2}\right)$. It hosts training facilities 
Table 1: List of alternative garages with parking and maintenance functionality. Distance: the distance between the alternative garage to BRT line $(\mathrm{km})$

\begin{tabular}{llll}
\hline Alternatives & Starting Date & Area $\left(\mathrm{m}^{2}\right)$ & Distance $(\mathrm{km})$ \\
\hline Ikitelli & 1986 & 196,322 & 20 \\
Edirnekapi & 1999 & 60 & 0.5 \\
Hasanpasa & 1984 & 33,862 & 1 \\
Anadolu & 1986 & 60 & 11.3 \\
Kagthane & 1995 & 60,004 & 5.8 \\
\hline
\end{tabular}

and a bus fleet control center. In terms of enough space, it is the most appropriate location for putting electric charging stations.

- Kagithane: Kagithane Garaji is located in the European part of Istanbul in a moderately large area. It mainly hosts CNG powered buses and has a CNG filling station. It also hosts a bus fleet control center. However, it does not have enough space for electric buses or electric charging stations.

- Edirnekapi: Edirnekapi garage is located in the European part of Istanbul and very close to the BRT route. It mainly hosts BRT buses and a BRT fleet control center. It also has enough space to locate electric buses and electric charging stations.

- Anadolu: Anadolu garage is located in the Asian part of Istanbul. It is far from the BRT line and mainly serves the bus services in the Asian part.

- Hasanpasa: Hasanpasa garage is located at the Asian end of the BRT route and hosts BRT buses. The main disadvantage of this garage is that it has the smallest garage area among all other garages. Figure 2 depicts all facilities for BRT buses.

\section{Preliminaries}

This section introduces the fundamentals of the techniques used in this work including Interval Type-2 fuzzy sets, fuzzy aggregation methods, and simulated 


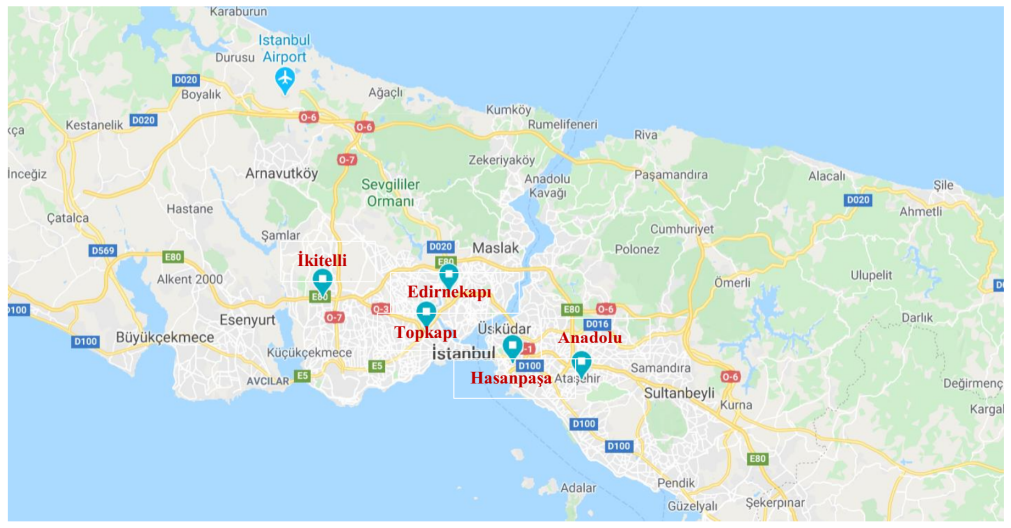

Figure 2: Five bus garages operated by IETT.

annealing metaheuristic.

\subsection{Interval Type-2 Fuzzy Sets}

For type-2 fuzzy systems, a membership function (the degree of membership) denoted as $\mu_{\tilde{A}}(x, u)$ characterised as fuzzy set $\tilde{A}$ where $x \in X$ in $\tilde{A}$ and $u \in$ $[0,1][38$. It is also shown as:

$$
\left.\tilde{A}=\left\{(x, u), \mu_{\tilde{A}}(x, u)\right) \mid \forall x \in X, \forall u \in[0,1]\right\}
$$

where $0 \leq \mu_{\tilde{A}}(x, u) \leq 1$. When all $\mu_{\tilde{A}}(x, u)=1$ for $\forall x \in X$ and $u \in[0,1]$, then $\tilde{A}$ is named as an interval type-2 fuzzy set 20 . It is denoted as:

$$
\tilde{A}=\int_{x \in X} \int_{u \in[0,1]} 1 /(x, u) \in[0,1]
$$

where $\iint$ denotes union over all admissible $\mathrm{x}$ and $\mathrm{u}$. For discrete universes of discourse, is replaced by $\sum$.

Although membership functions may have different shapes such as triangular, trapezoidal, Gaussian, etc., in this study, the trapezoidal interval type-2 fuzzy sets (IT2FSs) are used, where a membership function in the form of a trapezoid is denoted as $\tilde{A}_{i}$, shown as an example as follows [39:

$$
\begin{aligned}
\tilde{A}_{i}=\left(\tilde{A}_{i}^{U}, \tilde{A}_{i}^{L}\right)= & \left(\left(a_{i 1}^{u}, a_{i 2}^{u}, a_{i 3}^{u}, a_{i 4}^{u} ; h_{1}\left(\tilde{A}_{i}^{U}\right), h_{2}\left(\tilde{A}_{i}^{U}\right)\right),\right. \\
& \left(a_{i 1}^{l}, a_{i 2}^{l}, a_{i 3}^{l}, a_{i 4}^{l} ; h_{1}\left(\tilde{A}_{i}^{L}\right), h_{2}\left(\tilde{A}_{i}^{L}\right)\right)
\end{aligned}
$$


where $A_{i}^{U}$ and $A_{i}^{L}$ are type-1 fuzzy sets, $a_{i 1}^{u}, a_{i 2}^{u}, a_{i 3}^{u}, a_{i 4}^{u} ; a_{i 1}^{l}, a_{i 2}^{l}, a_{i 3}^{l}$ and $a_{i 4}^{l}$ are the reference points of the interval type-2 fuzzy set $\tilde{A}_{i}, H_{j}\left(\tilde{A}_{i}^{U}\right)$ denotes the membership value of the element $a_{i(j+1)}^{U}$ in the upper trapezoidal membership function $\tilde{A}_{i}^{u}, 1 \leq j \leq$ $2, H_{j}\left(\tilde{A}_{i}^{L}\right)$ denotes the membership value of the element $a_{i(j+1)}^{L}$ in the lower trapezoidal membership function $\tilde{A}_{i}^{L}, 1 \leq j \leq 2$. In Figure 3 the trapezoidal membership function for the linguistic term 'Medium' is demonstrated as an example.

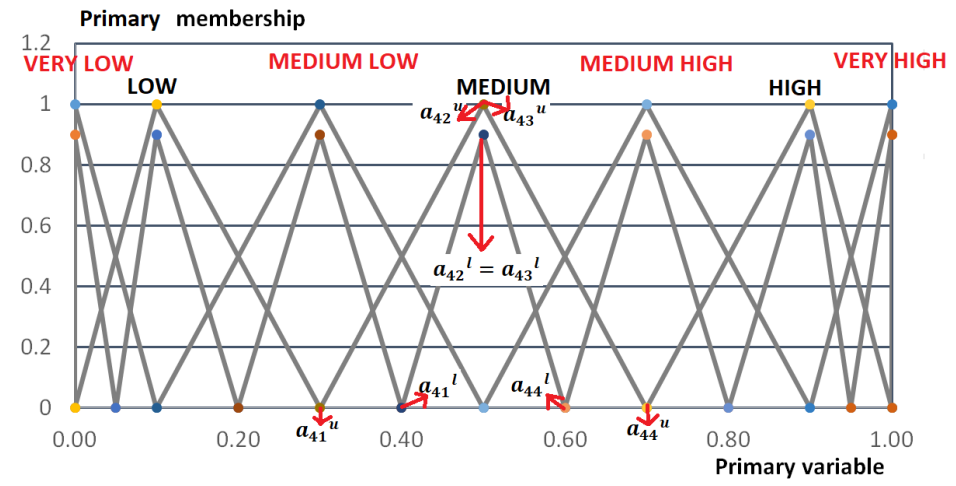

Figure 3: Illustration of IT2FSs

\subsection{Fuzzy aggregation methods}

The algebraic operations used in this study are addition and multiplication [40]. The addition of two fuzzy trapezoid membership functions $\tilde{A}_{1}$ and $\tilde{A}_{2}$ can be computed as in Equation 4.

$$
\begin{aligned}
\tilde{A}_{1} \oplus \tilde{A}_{2} & =\left(\tilde{A}_{1}^{U}, \tilde{A}_{1}^{L}\right) \oplus\left(\tilde{A}_{2}^{U}, \tilde{A}_{2}^{L}\right) \\
& =\left(\left(a_{11}^{u}+a_{21}^{u}, a_{12}^{u}+a_{22}^{u}, a_{13}^{u}+a_{23}^{u}, a_{14}^{u}+a_{24}^{u} ;\right.\right. \\
& \min \left(h_{1}\left(\tilde{A}_{1}^{U}\right), h_{1}\left(\tilde{A}_{2}^{U}\right)\right), \\
& \left.\min \left(h_{2}\left(\tilde{A}_{1}^{U}\right), h_{2}\left(\tilde{A}_{1}^{U}\right)\right)\right) \\
& \left(a_{11}^{l}+a_{21}^{l}, a_{12}^{l}+a_{22}^{l}, a_{13}^{l}+a_{23}^{l}, a_{14}^{l}+a_{24}^{l} ;\right. \\
& \left.\min \left(h_{1}\left(\tilde{A}_{1}^{L}\right), h_{1}\left(\tilde{A}_{2}^{L}\right)\right),\left(h_{2}\left(\tilde{A}_{1}^{L}\right), h_{2}\left(\tilde{A}_{2}^{L}\right)\right)\right)
\end{aligned}
$$


The multiplication of $\tilde{A}_{1}$ and $\tilde{A}_{2}$ can be computed as in Equation 5 .

$$
\begin{aligned}
\tilde{A}_{1} \otimes \tilde{A}_{2} & =\left(\tilde{A}_{1}^{U}, \tilde{A}_{1}^{L}\right) \otimes\left(\tilde{A}_{2}^{U}, \tilde{A}_{2}^{L}\right) \\
& =\left(\left(a_{11}^{u} \times a_{21}^{u}, a_{12}^{u} \times a_{22}^{u}, a_{13}^{u} \times a_{23}^{u}, a_{14}^{u} \times a_{24}^{u} ;\right.\right. \\
& \min \left(h_{1}\left(\tilde{A}_{1}^{U}\right), h_{1}\left(\tilde{A}_{2}^{U}\right)\right), \\
& \left.\min \left(h_{2}\left(\tilde{A}_{1}^{U}\right), h_{2}\left(\tilde{A}_{2}^{U}\right)\right)\right), \\
& \left(a_{11}^{l} \times a_{21}^{l}, a_{12}^{l} \times a_{22}^{l}, a_{13}^{l} \times a_{23}^{l}, a_{14}^{l} \times a_{24}^{l} ;\right. \\
& \left.\min \left(h_{1}\left(\tilde{A}_{1}^{L}\right), h_{1}\left(\tilde{A}_{2}^{L}\right)\right),\left(h_{2}\left(\tilde{A}_{1}^{L}\right), h_{2}\left(\tilde{A}_{2}^{L}\right)\right)\right) .
\end{aligned}
$$

\subsubsection{The linguistic weighted sum (LWS)}

In general, it has been known that linguistic values can be decomposed into the weighted sum of the linguistic values on distinct space 41. For instance, suppose that each sub-criterion is multiplied by its main criterion and it is done for all sub-criteria. In general, the score is defined as the weighted sum of the values obtained by each sub-criterion.

\subsubsection{The linguistic weighted average (LWA)}

$\mathrm{Wu}$ and Mendel 42 proposed the linguistic weighted average approach that can be used in distributed and hierarchical decision-making applications. The linguistic weighted average for interval type-2 fuzzy sets is explained as an extension of the fuzzy weighted average for type-1 fuzzy sets 43. For each alternative $a$ and main criterion $c$ pair, the LWA $\tilde{Y}_{a c}$ is computed as follows:

$$
\tilde{Y}_{a c}=\frac{\sum_{n=1}^{m_{c}} \tilde{X}_{a c n} \tilde{W}_{c n}}{\sum_{n=1}^{m_{c}} \tilde{W}_{c n}}
$$

where $\tilde{X}_{a c n}$ is the linguistic assessment of sub-criterion $n$ which is associated with the main criterion $c$ for alternative $a$ where $\tilde{W}_{c n}$ is the weight for sub-criterion $n . m_{c}$ is the number of each sub-criterion for each main criterion $c$ 43.

\subsection{Simulated Annealing (SA)}

Simulated Annealing is a well-known iterative metaheuristic inspired by the annealing process in the metallurgical industry [4, 45]. In order to achieve an approximate (near-optimal) solution for computationally difficult optimisation problems, SA algorithm as a single point search method has been generally preferred by researches as well 
as practitioners. A recent study shows that a tuned SA can be very effective to solve even an unseen problem [36]. SA starting form an initial candidate solution attempts to improve it in time by making changes to that solution at each iteration. The main feature of SA is that worsening solutions can be accepted by some probability which dynamically changes in time based on temperature and the change in the quality of the solution. Hence, while searching for the best solution to a problem instance, the quality of each candidate solution needs to be evaluated and this is achieved by using an objective function.

The pseudo-code of the SA approach used in this study is provided in Algorithm 1 . SA starts the search process with a randomly generated initial solution denoted as $S_{\text {Sol }}{ }_{\text {initial }}$ and an initial temperature $T_{\text {initial }}$. The objective function $f$ evaluates the initial solution and assigns the value to $Q_{\text {current }}$ at the beginning. Then the SA algorithm moves over the search space of solutions using a predefined neighbourhood/move operator, denoted as Create $_{\text {neighboringSol }}$ in line 6 of the Algorithm 1 . This move operator designed specifically for the problem is applied to the current solution, Sol current $_{\text {che }}$ generating a new solution, $S_{\text {new }}$ at each step. The difference of the objective values of $S o l_{\text {new }}$ and $S_{\text {colrent }}$ is computed as in line 7 and this value is denoted as $\Delta O b j$. Any improving new solution $(\Delta O b j<0)$ is directly accepted while a worsening solution is accepted with the probability of $e^{\Delta O b j / T}$, where $T$ is the current temperature (line 8), and the current solution is set to the new solution (line 9). The best solution could be lost as worsening solutions are accepted by SA, hence an explicit mechanism is needed to maintain/remember the best solution found so far (lines 12-14). Then the temperature is reduced using a cooling schedule at each step which reduces the probability of acceptance of worsening solutions in time. In this study, a geometric cooling schedule is used and $T$ is multiplied by a constant factor $0<C T<1$ (line 16). This iterative process of creating new solutions, evaluating them and accepting/rejecting them is repeated until a termination criteria, i.e., the maximum number of iterations or a final temperature of $T_{\min }$ is reached (line 5) [46].

\section{Proposed Approach}

In this study, we introduce an MCDM interval type-2 fuzzy approach combined with SA for selecting a garage considering the alternatives to place the electric charging stations. There are various methods to rank the bus garages for the final decision, and 


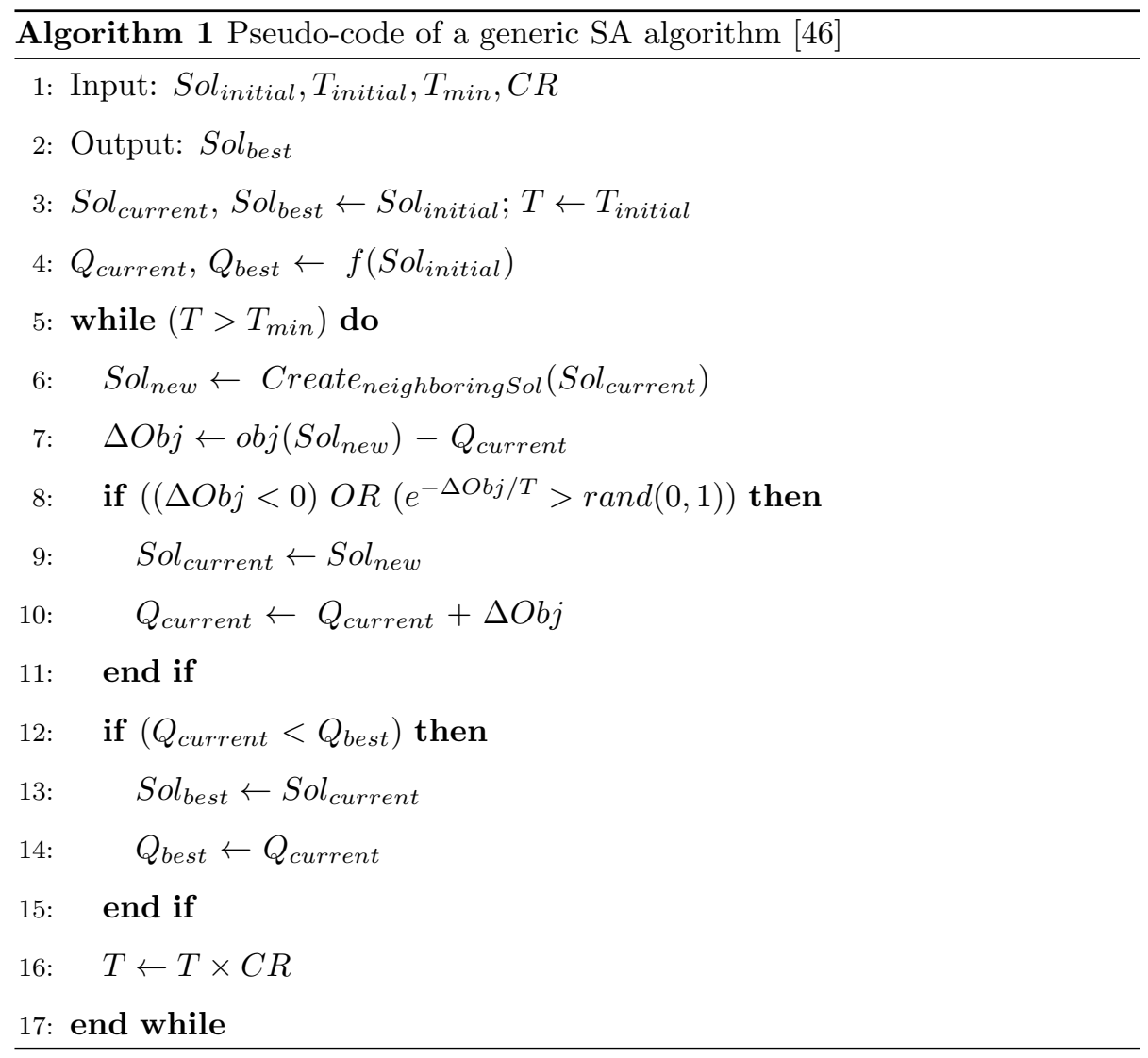

we tested two aggregation methods for ranking, namely the linguistic weighted sum and average. In this section, we explain the details of our overall approach starting with the specifics of the interval type-2 fuzzy membership functions, SA which is used to improve the membership functions and then covering the ranking methods.

\subsection{Fuzzy Membership Functions}

In this study, we consulted and did a survey with four decision-makers referred to as D1, D2, D3, and D4, working at IETT. A set of specific criteria are identified for the overall evaluation process of the alternative bus garages based on the extant literature and expert opinion as detailed in Section 2 .

Firstly, the decision-makers rated the importance of each of the 5 main criteria and 14 sub-criteria as in Table 6 and 7 respectively, using the linguistic terms 'Very Low' (VL), 'Low' (L), 'Medium Low' (ML), 'Medium' (M), 'Medium High' (MH), 'High' 
Table 2: Linguistic variables for evaluating each criterion [4], it also represents $\tilde{W}$ for LWA approach.

\begin{tabular}{l|llllll|llllll}
\hline Trapezoidal ITFSs & $a_{1}^{u}$ & $a_{2}^{u}$ & $a_{3}^{u}$ & $a_{4}^{u}$ & $h_{1}^{u}$ & $h_{2}^{u}$ & $a_{1}^{l}$ & $a_{2}^{l}$ & $a_{3}^{l}$ & $a_{4}^{l}$ & $h_{1}^{l}$ & $h_{2}^{l}$ \\
\hline Very low (VL) & 0 & 0 & 0 & 0.1 & 1 & 1 & 0 & 0 & 0 & 0.05 & 0.9 & 0.9 \\
Low (L) & 0 & 0.1 & 0.1 & 0.3 & 1 & 1 & 0.05 & 0.1 & 0.1 & 0.2 & 0.9 & 0.9 \\
Medium low (ML) & 0.1 & 0.3 & 0.3 & 0.5 & 1 & 1 & 0.2 & 0.3 & 0.3 & 0.4 & 0.9 & 0.9 \\
Medium (M) & 0.3 & 0.5 & 0.5 & 0.7 & 1 & 1 & 0.4 & 0.5 & 0.5 & 0.6 & 0.9 & 0.9 \\
Medium high (MH) & 0.5 & 0.7 & 0.7 & 0.9 & 1 & 1 & 0.6 & 0.7 & 0.7 & 0.8 & 0.9 & 0.9 \\
High (H) & 0.7 & 0.9 & 0.9 & 1 & 1 & 1 & 0.8 & 0.9 & 0.9 & 0.95 & 0.9 & 0.9 \\
Very high (VH) & 0.9 & 1 & 1 & 1 & 1 & 1 & 0.95 & 1 & 1 & 1 & 0.9 & 0.9 \\
\hline
\end{tabular}

Table 3: Linguistic variables for evaluating each alternative [47.

\begin{tabular}{l|cccccc|cccccc}
\hline Trapezoidal ITFSs & $a_{1}^{u}$ & $a_{2}^{u}$ & $a_{3}^{u}$ & $a_{4}^{u}$ & $h_{1}^{u}$ & $h_{2}^{u}$ & $a_{1}^{l}$ & $a_{2}^{l}$ & $a_{3}^{l}$ & $a_{4}^{l}$ & $h_{1}^{l}$ & $h_{2}^{l}$ \\
\hline Very poor (VP) & 0 & 0 & 0 & 1 & 1 & 1 & 0 & 0 & 0 & 0.5 & 0.9 & 0.9 \\
Poor (P) & 0 & 1 & 1 & 3 & 1 & 1 & 0.5 & 1 & 1 & 2 & 0.9 & 0.9 \\
Medium poor (MP) & 1 & 3 & 3 & 5 & 1 & 1 & 2 & 3 & 3 & 4 & 0.9 & 0.9 \\
Fair (F) & 3 & 5 & 5 & 7 & 1 & 1 & 4 & 5 & 5 & 6 & 0.9 & 0.9 \\
Medium good (MG) & 5 & 7 & 7 & 9 & 1 & 1 & 6 & 7 & 7 & 8 & 0.9 & 0.9 \\
Good (G) & 7 & 9 & 9 & 10 & 1 & 1 & 8 & 9 & 9 & 9.5 & 0.9 & 0.9 \\
Very good (VG) & 9 & 10 & 10 & 10 & 1 & 1 & 9.5 & 10 & 10 & 10 & 0.9 & 0.9 \\
\hline
\end{tabular}

(H), 'Very High' (VH). Table 2 shows the corresponding interval type-2 fuzzy sets for each term, specified using a real value between 0 and 1 . For a better understanding, the trapezoidal membership function for the linguistic term 'Medium' is demonstrated in Figure 3

After rating the importance of each criterion, the decision-makers provided a performance evaluation of each bus garage based on all criteria as illustrated in Table 9 using the linguistic terms Very Poor' (VP), Poor' (P), Medium Poor' (MP),Fair' (F), Medium Good' (MG), Good' (G), Very Good' (VG). Table 3 shows the associated interval type- 2 fuzzy sets for each term, specified using an integer value between 0 and 10. 


\subsection{Configuring fuzzy membership functions using $S A$}

We apply the SA approach as described in Section 3.3 to obtain the best configuration for the fuzzy membership functions to be used by the approach based on interval type-2 fuzzy sets. In this section, we provide the details of the SA approach, including the design of the problem domain-specific components, applied to the problem of bus garage selection to locate the electric charging stations for electric buses.

A real-valued candidate solution representation is used in SA. This is because each fuzzy membership function is designed as a trapezoid which can be uniquely represented using four real-valued parameters (see Section 3.1. Assuming that all type-2 fuzzy membership functions are known prior, a candidate solution is a 2-dimensional real-valued array that encodes the distances as in (val1, val2, val3, val4) to the reference points $\left(a_{1}^{l}, a_{2}^{l}, a_{3}^{l}, a_{4}^{l}\right)$ for each trapezoidal membership function for the importance weights. Hence, decoding a candidate solution yields a fixed fuzzy trapezoidal membership function for each linguistic term as in $\left(a_{1}^{l \prime}{ }^{\prime}=a_{1}^{l}-\right.$ val1, $a_{2}^{l \prime}=a_{2}^{l}, a_{3}^{l \prime}=a_{3}^{l}$, $a_{4}^{l \prime}=a_{1}^{l}+$ val4). Table 4 summarises the maximum possible distances for val1, val2, val3, and val4, calculated using $a_{1}^{l}-a_{1}^{u}, a_{2}^{l}-a_{2}^{u}, a_{3}^{l}-a_{3}^{u}$, and $a_{4}^{u}-a_{4}^{l}$, respectively. For illustrative purposes, let's use 'Medium (M)' membership set in Figure 4(a) characterised by $\left(a_{1}^{l}=0.4, a_{2}^{l}=0.5, a_{3}^{l}=0.5, a_{4}^{l}=0.6\right)$. Assuming that we have a candidate solution represented by four parameter values that are distances to the reference points (val1 $=0.07$, val $2=0$, val $3=0$ and val $4=0.017)$, then this solution decodes into the fuzzy membership function as illustrated in Figure 4 (b), the new lower bound values moving from 0.4 to 0.33 for $a_{1}^{l}$ and 0.6 to 0.617 for $a_{4}^{l}$ while $a_{2}^{l}$ and $a_{3}^{l}$ maintain the same values. Parameterising the membership functions this way enables the use of optimisation methods for detecting the best (optimal) parameter settings (configuration of membership functions) and improving the fuzzy system at the end based on a certain objective. We use Simulated Annealing for this purpose. 


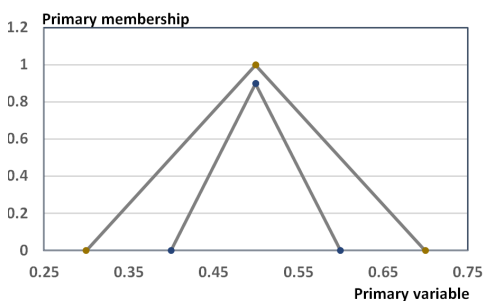

(a)

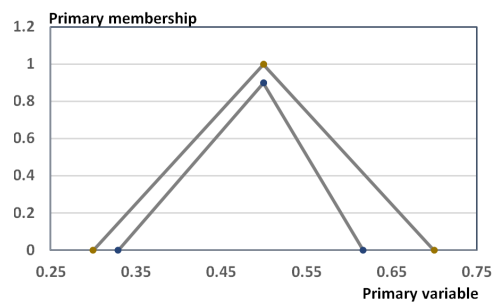

(b)

Figure 4: Illustration of (a) four parameters $\left(a_{1}^{l}=0.4, a_{2}^{l}=0.5, a_{3}^{l}=0.5, a_{4}^{l}=0.6\right)$ characterising the 'Medium' trapezoidal membership function for the importance of criteria, and (b) the resultant 'Medium' trapezoidal membership function after decoding a candidate solution with the given distances of $($ val $1=0.07$, val $2=0$, val $3=0$ and val4 $=0.017)$ into $\left(a_{1}^{l \prime}=0.33, a_{2}^{l \prime}=0.5\right.$, $\left.a_{3}^{l \prime}=0.5, a_{4}^{l \prime}=0.617\right)$.

Table 4: The maximum distances between upper and lower bounds of the trapezoids representing linguistic variables in Table 2

\begin{tabular}{lcccc}
\hline Linguistic variables & $\max \{\operatorname{val1}\}$ & $\max \{\operatorname{val} 2\}$ & $\max \{\operatorname{val} 3\}$ & $\max \{\operatorname{val} 4\}$ \\
\hline Very low $(\mathrm{VL})$ & 0 & 0 & 0 & 0.05 \\
Low $(\mathrm{L})$ & 0.05 & 0 & 0 & 0.1 \\
Medium low (ML) & 0.1 & 0 & 0 & 0.1 \\
Medium (M) & 0.1 & 0 & 0 & 0.1 \\
Medium high (MH) & 0.1 & 0 & 0 & 0.1 \\
High (H) & 0.1 & 0 & 0 & 0.05 \\
Very high $(\mathrm{VH})$ & 0.05 & 0 & 0 & 0 \\
\hline
\end{tabular}

In this study, the Root Mean Square Error (RMSE) is used as the objective function as defined below:

$$
R M S E=\sqrt{\frac{1}{n} \Sigma_{i=1}^{n}\left(f_{i}-f_{i}^{*}\right)^{2}}
$$

where $f_{i}$ represents the value found by SA while $f_{i}^{*}$ demonstrated the maximum value for each alternative.

First, the maximum value for the first alternative is calculated assigning each criterion 'very high' for the first one and 'very low' for the rest. The maximum value of each alternative is found using the same way. Then RMSE is calculated using 
the difference between maximum values and values achieved by a single SA run. In Equation 7. $f_{i}$ shows the maximum value assigned for $i^{\text {th }}$ alternative and $f_{i}^{*}$ represents the value found by SA for $i^{t h}$ alternative. Therefore, the objective is to minimize RMSE.

In this study, Algorithm 1 is employed starting with a randomly created initial solution. The move operator Create $_{n e i g h b o r i n g S o l}$ perturbs randomly selected array entries of a given candidate solution, creating a new one. Each entry is randomly perturbed to a valid value considering the maximum distance for the relevant fuzzy set. This perturbative move operator has a discrete strength parameter taking an integer value in $[1,10]$, indicating the strength of the perturbation, that is, how many elements are modified in a given solution. A simple reinforcement learning mechanism 46] is used to adaptively control the strength setting of the move operator. A utility score for each of the 10 settings for the strength parameter is maintained. Initially, all scores are set to the same value. After applying the move operator with a particular strength setting, if an improved solution is obtained then the score for this setting is increased by one, otherwise, it is decreased by one. At each step, the strength setting with the highest score is selected to be used with the move operator. Random choice is the tie-breaking strategy if there are multiple settings with the highest score. More on this method can be found in Turk et al. [46]. Cooling rate as shown $C R$ in Algorithm 1 is computed as in Equation 8 .

$$
C R=e^{\log \left(\left(T_{\text {termination }} / T\right) / \text { MaxIts }\right)}
$$

where $T, T_{\text {termination }}$ and MaxIts represent the 'current temperature', 'termination temperature' and 'number of maximum iterations', respectively.

\subsection{Linguistic weighted sum (LWS) approach}

This approach consists of six steps explained as follows:

Step 1: Decision makers decided main and sub-criteria as shown in Table 5 . The importance of each criterion is determined by four decision-makers as shown in Table 6 for main criteria and Table 7 for sub-criteria, respectively. For example, the first decision maker (DM1) defines the importance of the first main criterion (MC1) as 'Very High' (VH) while the second decision maker (DM2) assigns MC1 as 'High' (H). 
Table 5: List of main and sub-criteria

\begin{tabular}{lll}
\hline Main & Symbol & Sub-criteria \\
\hline Economy (M1) & C1 & Investment return period \\
& C2 & Revenue potential \\
& C3 & Closeness to power grid \\
& C4 & Reliability of electricity power \\
Energy (M2) & C5 & Distance from heavy electricity consumption \\
& C6 & Availability of renewable energy resources \\
& C7 & Closeness to BRT or Interchange stations \\
Service Area (M3) & C8 & Traffic congestion \\
& C9 & Closeness to bus routes \\
& C10 & Topographical properties (high slope) \\
& C11 & Availability of garage space \\
Garage Capacity (M4) & C12 & Expandability \\
& C13 & Maintenance capability \\
Safety (M5) & C14 & Security infrastructure \\
\hline
\end{tabular}

Step 2: Each main criterion and its corresponding sub-criterion are multiplied in order to calculate interval type- 2 sets of 14 criteria. In Table 8 , these weighted fuzzy sets are illustrated using symbol from C1 to C14. Let us compute C1 for DM1 to show how to convert the linguistic terms to fuzzy numbers as an example:

$$
\begin{array}{r}
C 1=(0.9,1.0,1.0,1.0 ; 1.0,1.0),(0.95,1.0,1.0,1.0 ; 0.9,0.9) \\
\otimes(0.7,0.9,0.9,1.0 ; 1.0,1.0),(0.8,0.9,0.9,0.95 ; 0.9,0.9) \\
=(0.63,0.9,0.9,1.0 ; 1.0,1.0),(0.76,0.9,0.9,0.95 ; 0.9,0.9)
\end{array}
$$

The rest is computed in the same way for the other three decision-makers and the average of these values is taken as the importance weight of $\mathrm{C} 1$ and shown in Table 8 .

Step 3: The linguistic terms of the importance of each alternative are defined in Table 9. These terms are converted into interval type-2 fuzzy sets. 
Table 6: Importance of main criteria according to decision-makers

\begin{tabular}{lccccc}
\hline Decision Makers & MC1 & MC2 & MC3 & MC4 & MC5 \\
\hline DM1 & VH & H & MH & H & M \\
DM2 & H & VH & H & VH & M \\
DM3 & $\mathrm{H}$ & VH & VH & H & ML \\
DM4 & VH & VH & MH & MH & MH \\
\hline
\end{tabular}

Table 7: Importance of sub-criteria according to decision-makers

\begin{tabular}{llllllll}
\hline Decision makers & $\mathrm{C} 1$ & $\mathrm{C} 2$ & $\mathrm{C} 3$ & $\mathrm{C} 4$ & $\mathrm{C} 5$ & $\mathrm{C} 6$ & $\mathrm{C} 7$ \\
\hline DM1 & $\mathrm{H}$ & $\mathrm{H}$ & $\mathrm{MH}$ & $\mathrm{MH}$ & $\mathrm{ML}$ & $\mathrm{M}$ & $\mathrm{VH}$ \\
DM2 & $\mathrm{MH}$ & $\mathrm{H}$ & $\mathrm{H}$ & $\mathrm{VH}$ & $\mathrm{M}$ & $\mathrm{L}$ & $\mathrm{H}$ \\
DM3 & $\mathrm{H}$ & $\mathrm{VH}$ & $\mathrm{H}$ & $\mathrm{MH}$ & $\mathrm{M}$ & $\mathrm{MH}$ & $\mathrm{MH}$ \\
DM4 & $\mathrm{VH}$ & $\mathrm{MH}$ & $\mathrm{VH}$ & $\mathrm{H}$ & $\mathrm{MH}$ & $\mathrm{MH}$ & $\mathrm{H}$ \\
\hline Decision makers & $\mathrm{C} 8$ & $\mathrm{C} 9$ & $\mathrm{C} 10$ & $\mathrm{C} 11$ & $\mathrm{C} 12$ & $\mathrm{C} 13$ & $\mathrm{C} 14$ \\
\hline DM1 & $\mathrm{MH}$ & $\mathrm{H}$ & $\mathrm{M}$ & $\mathrm{H}$ & $\mathrm{M}$ & $\mathrm{M}$ & $\mathrm{MH}$ \\
DM2 & $\mathrm{MH}$ & $\mathrm{VH}$ & $\mathrm{MH}$ & $\mathrm{VH}$ & $\mathrm{H}$ & $\mathrm{MH}$ & $\mathrm{ML}$ \\
DM3 & $\mathrm{M}$ & $\mathrm{VH}$ & $\mathrm{ML}$ & $\mathrm{MH}$ & $\mathrm{M}$ & $\mathrm{H}$ & $\mathrm{ML}$ \\
DM4 & $\mathrm{M}$ & $\mathrm{H}$ & $\mathrm{M}$ & $\mathrm{H}$ & $\mathrm{ML}$ & $\mathrm{MH}$ & $\mathrm{L}$ \\
\hline
\end{tabular}

Step 4: The aggregate fuzzy sets for each alternative is calculated by multiplying interval type-2 fuzzy sets for performance by fuzzy importance weights of criteria.

Step 5: The fuzzy set values for each alternative is converted into crisp values using Centroid type-reduction and defuzzification methods.

Step 6: By considering the crisp values that we obtained, the alternatives are ranked.

\subsection{Linguistic weighted average (LWA) approach}

For the LWA approach, all steps except Step 2 are carried out in the same manner as described for the linguistic weighted sum approach as in the previous subsection. In Step 2, the main difference is the way of "computing with words' [16. Each criterion is calculated based on its sub-criteria using formulas 
Table 8: The weighted trapezoidal interval type-2 sets for criteria

\begin{tabular}{cc}
\hline Criterion & Interval type-2 fuzzy sets \\
\hline C1 & $(0.57,0.59,0.84,0.98 ; 1.0,1.0),(0.70,0.84,0.84,0.90 ; 0.9,0.9)$ \\
C2 & $(0.55,0.83,0.83,0.98 ; 1.0,1.0),(0.68,0.83,0.83,0.90 ; 0.9,0.9)$ \\
C3 & $(0.61,0.86,0.86,0.98 ; 1.0,1.0),(0.73,0.86,0.86,0.89 ; 0.9,0.9)$ \\
C4 & $(0.56,0.81,0.81,0.95 ; 1.0,1.0),(0.68,0.81,0.81,0.88 ; 0.9,0.9)$ \\
C5 & $(0.27,0.49,0.49,0.70 ; 1.0,1.0),(0.37,0.49,0.49,0.60 ; 0.9,0.9)$ \\
C6 & $(0.28,0.49,0.49,0.70 ; 1.0,1.0),(0.38,0.49,0.49,0.60 ; 0.9,0.9)$ \\
C7 & $(0.44,0.71,0.71,0.93 ; 1.0,1.0),(0.57,0.71,0.71,0.82 ; 0.9,0.9)$ \\
C8 & $(0.26,0.49,0.49,0.76 ; 1.0,1.0),(0.37,0.49,0.49,0.62 ; 0.9,0.9)$ \\
C9 & $(0.54,0.79,0.79,0.95 ; 1.0,1.0),(0.66,0.79,0.79,0.87 ; 0.9,0.9)$ \\
C10 & $(0.17,0.41,0.41,0.67 ; 1.0,1.0),(0.29,0.41,0.41,0.53 ; 0.9,0.9)$ \\
C11 & $(0.50,0.77,0.77,0.95 ; 1.0,1.0),(0.63,0.77,0.77,0.86 ; 0.9,0.9)$ \\
C12 & $(0.28,0.50,0.50,0.71 ; 1.0,1.0),(0.38,0.50,0.50,0.60 ; 0.9,0.9)$ \\
C13 & $(0.35,0.61,0.61,0.85 ; 1.0,1.0),(0.47,0.61,0.61,0.73 ; 0.9,0.9)$ \\
\hline & $(0.05,0.17,0.17,0.38 ; 1.0,1.0),(0.10,0.17,0.17,0.26 ; 0.9,0.9)$ \\
\hline
\end{tabular}

shown in Section 3.2 .2 . All alternatives are evaluated in the same manner as explained Section 4.3 .

\section{Experimental Results}

The experiments are performed on an Intel i3 Windows 7 machine $(2.4 \mathrm{GHz})$ with 6 GB RAM.

\subsection{Results from the Interval Type-2 Fuzzy Sets}

The results from the interval type-2 fuzzy sets using LWS and LWA approaches are summarised in Table 10 , providing the ranking of each alternative. 
Table 9: Performance evaluation of decision-makers

\begin{tabular}{|c|c|c|c|c|c|c|c|c|}
\hline Alternatives & $\begin{array}{l}\text { Decision } \\
\text { Makers }\end{array}$ & $\mathrm{C} 1$ & $\mathrm{C} 2$ & C3 & $\mathrm{C} 4$ & $\mathrm{C} 5$ & $\mathrm{C} 6$ & $\mathrm{C} 7$ \\
\hline \multirow{4}{*}{ Ikitelli } & DM1 & MG & $\mathrm{F}$ & MG & G & VG & $\mathrm{F}$ & $\mathrm{F}$ \\
\hline & DM2 & G & MG & G & G & G & MG & MG \\
\hline & DM3 & $\mathrm{P}$ & MP & $\mathrm{F}$ & MG & $\mathrm{F}$ & $\mathrm{P}$ & $\mathrm{F}$ \\
\hline & DM4 & MP & MG & $\mathrm{P}$ & G & MG & $\mathrm{F}$ & MP \\
\hline \multirow{4}{*}{ Edirnekapi } & DM1 & G & G & VG & $\mathrm{F}$ & G & G & VG \\
\hline & DM2 & VG & G & G & G & G & MG & VG \\
\hline & DM3 & VG & VG & G & VG & $\mathrm{P}$ & G & VG \\
\hline & DM4 & G & VG & G & MG & $\mathrm{P}$ & MG & VG \\
\hline \multirow{4}{*}{ Hasanpasa } & DM1 & G & MG & $\mathrm{F}$ & $\mathrm{F}$ & $\mathrm{P}$ & $\mathrm{F}$ & VG \\
\hline & DM2 & MG & G & MP & MG & $\mathrm{VP}$ & G & G \\
\hline & DM3 & MG & G & G & MG & $\mathrm{P}$ & MG & VG \\
\hline & DM4 & G & MG & $\mathrm{P}$ & G & MP & G & $\mathrm{V}$ \\
\hline \multirow{4}{*}{ Anadolu } & DM1 & $\mathrm{F}$ & $\mathrm{P}$ & $\mathrm{F}$ & $\mathrm{F}$ & F & VP & VP \\
\hline & DM2 & MP & MP & $\mathrm{P}$ & MG & MG & $\mathrm{P}$ & $\mathrm{VP}$ \\
\hline & DM3 & VP & $\mathrm{P}$ & MP & MG & VG & $\mathrm{P}$ & $\mathrm{P}$ \\
\hline & DM4 & $\mathrm{P}$ & $\mathrm{F}$ & $\mathrm{P}$ & G & G & MG & MP \\
\hline \multirow{4}{*}{ Kagithane } & DM1 & $\mathrm{F}$ & MG & $\mathrm{F}$ & G & P & VG & $\mathrm{F}$ \\
\hline & DM2 & MG & MG & F & MP & $\mathrm{P}$ & VG & MP \\
\hline & DM3 & MP & MG & G & MG & MP & VG & G \\
\hline & DM4 & $\mathrm{F}$ & MP & $\mathrm{P}$ & G & $\mathrm{P}$ & VG & MG \\
\hline \multirow{2}{*}{ Alternatives } & Decision & $\mathrm{C} 8$ & $\mathrm{C} 9$ & $\mathrm{C} 10$ & $\mathrm{C} 11$ & C12 & $\mathrm{C} 13$ & C14 \\
\hline & Makers & & & & & & & \\
\hline \multirow{4}{*}{ Ikitelli } & DM1 & MG & G & $\mathrm{F}$ & VG & G & G & G \\
\hline & DM2 & G & G & MP & VG & MG & VG & G \\
\hline & DM3 & $\mathrm{F}$ & MP & F & VG & G & VG & G \\
\hline & DM4 & G & P & F & G & MG & G & VG \\
\hline \multirow{4}{*}{ Edirnekapi } & DM1 & $\mathrm{F}$ & F & $\mathrm{P}$ & $\mathrm{F}$ & G & VG & G \\
\hline & DM2 & MP & MP & $\mathrm{VP}$ & MG & VG & VG & VG \\
\hline & DM3 & $\mathrm{P}$ & $\mathrm{VG}$ & $\mathrm{P}$ & MG & VG & G & VG \\
\hline & DM4 & VP & G & $\mathrm{P}$ & $\mathrm{F}$ & VG & VG & VG \\
\hline \multirow{4}{*}{ Hasanpasa } & DM1 & G & G & MP & VP & $\mathrm{P}$ & $\mathrm{F}$ & G \\
\hline & DM2 & VG & G & $\mathrm{P}$ & $\mathrm{P}$ & VP & G & G \\
\hline & DM3 & $\mathrm{F}$ & G & $\mathrm{P}$ & P & P & MG & G \\
\hline & DM4 & MG & MG & VP & MP & $\mathrm{P}$ & F & G \\
\hline \multirow{4}{*}{ Anadolu } & DM1 & $\mathrm{P}$ & $\mathrm{F}$ & $\mathrm{F}$ & G & G & G & MG \\
\hline & DM2 & VP & MG & MP & VG & F & F & VG \\
\hline & DM3 & VG & $\mathrm{F}$ & MP & F & MG & MP & VG \\
\hline & DM4 & VG & MP & $\mathrm{F}$ & MG & MG & G & G \\
\hline \multirow{4}{*}{ Kagithane } & DM1 & F & MG & G & F & P & G & G \\
\hline & DM2 & MG & $\mathrm{VG}$ & VG & $\mathrm{P}$ & MP & G & VG \\
\hline & DM3 & G & MG & VG & $\mathrm{F}$ & F & G & G \\
\hline & DM4 & MG & $\mathrm{F}$ & VG & MP & MG & G & G \\
\hline
\end{tabular}

Both approaches yielded the same ranking for the alternatives. They show that 
Ikitelli is the best garage alternative for placing the electric charging stations with the crisp scores of 39.56 and 0.889 for the LWS and LWA approaches, respectively. The worst alternative is Hasanpasa with the crisp scores of 24.66 and 0.554 for the LWS and LWA approaches, respectively.

Table 10: Found Scores for Alternatives

\begin{tabular}{lcccc}
\hline \multirow{2}{*}{ Alternatives } & \multicolumn{2}{c}{ Score of LWS } & \multicolumn{2}{c}{ Score of LWA } \\
& Crisp Scores & Rank & Crisp Scores & Rank \\
\hline Ikitelli (A1) & 39.56 & 1 & 0.889 & 1 \\
Edirnekapi (A2) & 36.66 & 2 & 0.824 & 2 \\
Hasanpasa (A3) & 24.66 & 5 & 0.554 & 5 \\
Anadolu (A4) & 32.68 & 3 & 0.734 & 3 \\
Kagithane (A5) & 28.79 & 4 & 0.647 & 4 \\
\hline
\end{tabular}

\subsection{Simulated Annealing Experiments}

To investigate the impact of uncertainties involved in the linguistic evaluation of bus garages, this approach has been extended in an attempt to improve the results by using SA.

The main goal of this part of the study is to find out the best configuration for fuzzy sets representing the importance of criteria to make it capable to cope with the linguistic uncertainty better than fuzzy sets determined by decisionmakers at the beginning. The question of whether interval type-2 fuzzy systems can enhance the ability to handle information has been tackled within different fuzzy sets.

Each experiment is repeated for 30 trials generating 30 different solutions, each from a single SA run. In the SA algorithm, the cooling schedule is geometric which provides a slow and consistent reduction of temperature throughout a run, and for each run, the cooling rate is computed using Equation 8 . 


\subsubsection{Parameter Tuning of $S A$}

SA has three control parameters: $T_{\text {initial }}, T_{\text {termination }}$ and MaxIts as described in Section 3.3 . It is a known phenomenon that tuning a metaheuristic is likely to improve its performance. Hence, initial experiments are conducted for obtaining the best settings for those SA parameters. Identifying the best parameter setting for $\mathrm{SA}$, the $L^{9}$ Taguchi orthogonal arrays design [48] is used to tune its parameters based on a factorial design. Each of the nine parameter settings as provided in Table 11 is tested. The best SA configuration is detected based on the main effects as illustrated in Figure 5 , which is attained as 10,000 for $P 1,5 \times 10^{-6}$ for $P 2$, and $1,000,000$ for $P 3$. Validation is carried out testing the SA with those parameter settings and based on the RMSE scores, the tuned SA outperforms all the other settings as presented in Table 11 with the lowest RMSE score of 17.4308. Thus, this best parameter setting is fixed for the algorithm in the remaining experiments.

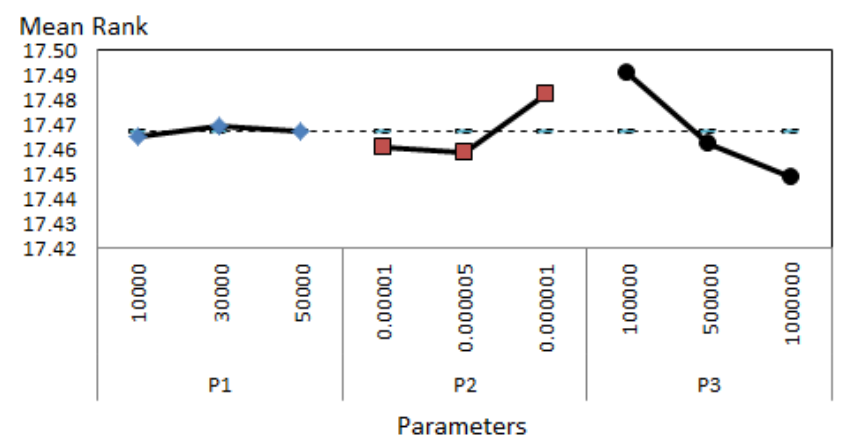

Figure 5: Main effects plot based on the RMSE scores running SA with different parameter settings, P1: Initial temperature, P2: Absolute temperature, P3: Maximum Evaluation. 
Table 11: RMSE scores obtained after running SA with each particular parameter configuration (Conf.).

\begin{tabular}{ccccc}
\hline Conf.ID & $T_{\text {initial }}$ & $T_{\text {termination }}$ & MaxIts & RMSE \\
\hline 1 & 10,000 & $1 \times 10^{-5}$ & 100,000 & 17.4792 \\
2 & 10,000 & $5 \times 10^{-6}$ & 500,000 & 17.4564 \\
3 & 10,000 & $1 \times 10^{-6}$ & $1,000,000$ & 17.4599 \\
4 & 30,000 & $1 \times 10^{-5}$ & 500,000 & 17.4564 \\
5 & 30,000 & $5 \times 10^{-6}$ & $1,000,000$ & 17.4399 \\
6 & 30,000 & $1 \times 10^{-6}$ & 100,000 & 17.5128 \\
7 & 50,000 & $1 \times 10^{-5}$ & $1,000,000$ & 17.4467 \\
8 & 50,000 & $5 \times 10^{-6}$ & 100,000 & 17.4800 \\
9 & 50,000 & $1 \times 10^{-6}$ & 500,000 & 17.4749 \\
\hline
\end{tabular}

\subsubsection{Analysing the Behavior of $S A$}

As for the behaviour of the SA during the search process, Figure 6 provides the progress-plot of mean RMSE of the current solution in time (based on iteration) averaged over 30 runs for ID1 for two of the aggregation approaches as an example. The RMSE score fluctuates radically during the initial phases of the search process. This denotes that large moves are accepted initially as expected, and this behaviour settles down in time and the algorithm seems to reach a plateau as expected.

\subsection{Results from the Proposed Approach}

The experiments are repeated for 30 trials with the best SA settings for both of the aggregation approaches. Each solution from SA is used to generate new fuzzy sets for the importance of criteria. 
Table 12: The widths of fuzzy sets for the importance of criteria for each solution generated by SA when the linguistic weighted sum is used. Each solution is reported along with the corresponding RMSE score and rank, where ID\#: solution ID, $a_{1}^{l}$ : Lower bound - first value, $a_{4}^{l}$ : Lower bound - forth value. The top 3 and worst solutions' RMSE scores and ranks are highlighted in bold.

\begin{tabular}{|c|c|c|c|c|c|c|c|c|c|c|c|c|c|}
\hline & $a_{1}^{l}$ & $a_{4}^{l}$ & $a_{1}^{l}$ & $a_{4}^{l}$ & $a_{1}^{l}$ & $a_{4}^{l}$ & & $a_{1}^{l}$ & $a_{4}^{l}$ & $a_{1}^{l}$ & $a_{4}^{l}$ & $a_{1}^{l}$ & $a_{4}^{l}$ \\
\hline LT & \multicolumn{2}{|c|}{ ID1 } & \multicolumn{2}{|c|}{ ID6 } & \multicolumn{2}{|c|}{ ID11 } & LT & \multicolumn{2}{|c|}{ ID16 } & \multicolumn{2}{|c|}{ ID21 } & \multicolumn{2}{|c|}{ ID26 } \\
\hline VL & 0 & 0.05836 & 0 & 0.08276 & 0 & 0.07915 & $\mathrm{VL}$ & 0 & 0.06706 & 0 & 0.07156 & 0 & 0.08782 \\
\hline L & 0.03723 & 0.23247 & 0.02223 & 0.24425 & 0.0052 & 0.29398 & L & 0.04636 & 0.24606 & 0.00192 & 0.26809 & 0.0413 & 0.21112 \\
\hline ML & 0.10015 & 0.46082 & 0.17039 & 0.46273 & 0.18723 & 0.41967 & ML & 0.11348 & 0.45435 & 0.1293 & 0.41685 & 0.16749 & 0.46626 \\
\hline M & 0.34473 & 0.63888 & 0.33439 & 0.63706 & 0.38193 & 0.65788 & M & 0.31516 & 0.6139 & 0.39887 & 0.68545 & 0.30181 & 0.68409 \\
\hline MH & 0.51386 & 0.87788 & 0.56944 & 0.82285 & 0.58952 & 0.86163 & $\mathrm{MH}$ & 0.54423 & 0.87498 & 0.52734 & 0.82102 & 0.54038 & 0.89684 \\
\hline $\mathrm{H}$ & 0.72363 & 0.9587 & 0.7541 & 0.98095 & 0.73024 & 0.95502 & $\mathrm{H}$ & 0.78975 & 0.9787 & 0.78567 & 0.98808 & 0.75818 & 0.97417 \\
\hline $\mathrm{VH}$ & 0.93333 & 1 & 0.92346 & 1 & 0.90414 & 1 & $\mathrm{VH}$ & 0.91707 & 1 & 0.90365 & 1 & 0.92318 & 1 \\
\hline RMSE & 17.4405 & & 17.4462 & & 17.4571 & & RMSE & 17.4344 & & 17.4803 & & 17.4314 & \\
\hline \multirow[t]{2}{*}{ Rank } & 11 & & 13 & & 22 & & Rank & 6 & & 30 & & 4 & \\
\hline & \multicolumn{2}{|c|}{ ID2 } & \multicolumn{2}{|c|}{ ID7 } & \multicolumn{2}{|c|}{ ID12 } & & \multicolumn{2}{|c|}{ ID177 } & \multicolumn{2}{|c|}{ ID22 } & \multicolumn{2}{|c|}{ ID27 } \\
\hline VL & 0 & 0.07142 & 0 & 0.06834 & 0 & 0.07871 & VL & 0 & 0.08828 & 0 & 0.07278 & 0 & 0.06178 \\
\hline L & 0.04049 & 0.2325 & 0.00252 & 0.29371 & 0.01397 & 0.27183 & L & 0.03726 & 0.29648 & 0.03004 & 0.24235 & 0.02399 & 0.28227 \\
\hline ML & 0.15341 & 0.49908 & 0.13785 & 0.49129 & 0.15269 & 0.49048 & ML & 0.17367 & 0.42086 & 0.11437 & 0.47455 & 0.12042 & 0.48231 \\
\hline M & 0.34811 & 0.67832 & 0.31235 & 0.68949 & 0.33016 & 0.6446 & M & 0.36903 & 0.60686 & 0.39255 & 0.62412 & 0.34525 & 0.65792 \\
\hline MH & 0.54543 & 0.86168 & 0.56592 & 0.8024 & 0.54784 & 0.81556 & $\mathrm{MH}$ & 0.50942 & 0.81904 & 0.50637 & 0.85585 & 0.56732 & 0.8432 \\
\hline $\mathrm{H}$ & 0.71211 & 0.97854 & 0.7636 & 0.98243 & 0.72075 & 0.99109 & $\mathrm{H}$ & 0.74577 & 0.99004 & 0.75234 & 0.96415 & 0.77377 & 0.99852 \\
\hline $\mathrm{VH}$ & 0.9024 & 1 & 0.90237 & 1 & 0.90977 & 1 & $\mathrm{VH}$ & 0.90237 & 1 & 0.92546 & 1 & 0.91781 & 1 \\
\hline RMSE & 17.4758 & & 17.4569 & & 17.4695 & & RMSE & 17.4620 & & 17.4267 & & 17.4329 & \\
\hline Rank & 29 & & 20 & & 27 & & Rank & 24 & & 2 & & 5 & \\
\hline & \multicolumn{2}{|c|}{ ID3 } & \multicolumn{2}{|c|}{ ID8 } & \multicolumn{2}{|c|}{ ID13 } & & \multicolumn{2}{|c|}{ ID18 } & & & ID & \\
\hline VL & 0 & 0.06471 & 0 & 0.07054 & 0 & 0.09973 & VL & 0 & 0.08345 & 0 & 0.07907 & 0 & 0.05363 \\
\hline L & 0.00581 & 0.25509 & 0.00449 & 0.25838 & 0.02306 & 0.25347 & $\mathrm{~L}$ & 0.02809 & 0.22826 & 0.02038 & 0.24686 & 0.0484 & 0.27091 \\
\hline ML & 0.16066 & 0.41482 & 0.10292 & 0.46336 & 0.18163 & 0.43584 & ML & 0.10047 & 0.45658 & 0.16177 & 0.48524 & 0.11269 & 0.42754 \\
\hline M & 0.34916 & 0.68507 & 0.38877 & 0.65459 & 0.38612 & 0.65551 & M & 0.31662 & 0.67918 & 0.31267 & 0.65832 & 0.3096 & 0.62875 \\
\hline MH & 0.57888 & 0.81423 & 0.5546 & 0.88908 & 0.50505 & 0.86849 & $\mathrm{MH}$ & 0.58294 & 0.80202 & 0.58489 & 0.84826 & 0.59046 & 0.85061 \\
\hline $\mathrm{H}$ & 0.72294 & 0.99959 & 0.76 & 0.99593 & 0.70981 & 0.95532 & $\mathrm{H}$ & 0.76615 & 0.99878 & 0.79723 & 0.98759 & 0.72032 & 0.97482 \\
\hline $\mathrm{VH}$ & 0.92401 & 1 & 0.93836 & 1 & 0.92023 & 1 & $\mathrm{VH}$ & 0.90285 & 1 & 0.9235 & 1 & 0.92623 & 1 \\
\hline RMSE & 17.4624 & & 17.4582 & & 17.4487 & & RMSE & 17.4407 & & 17.4510 & & 17.4381 & \\
\hline Rank & 25 & & 23 & & 15 & & Rank & 12 & & 16 & & 10 & \\
\hline & & 24 & & 99 & II & & & & & & & IL & 29 \\
\hline VL & 0 & 0.08805 & 0 & 0.09234 & 0 & 0.07728 & VL & 0 & 0.08988 & 0 & 0.08071 & 0 & 0.07792 \\
\hline $\mathrm{L}$ & 0.0264 & 0.22736 & 0.04474 & 0.20775 & 0.00185 & 0.27067 & $\mathrm{~L}$ & 0.01096 & 0.25507 & 0.03218 & 0.26899 & 0.03928 & 0.26156 \\
\hline ML & 0.1887 & 0.45033 & 0.13031 & 0.43659 & 0.15824 & 0.41003 & ML & 0.16675 & 0.46812 & 0.14007 & 0.43315 & 0.17859 & 0.45037 \\
\hline M & 0.38135 & 0.64086 & 0.38392 & 0.67858 & 0.36314 & 0.6425 & M & 0.35182 & 0.65345 & 0.30407 & 0.60249 & 0.33293 & 0.61343 \\
\hline MH & 0.53871 & 0.87719 & 0.54973 & 0.85516 & 0.57152 & 0.8905 & $\mathrm{MH}$ & 0.52898 & 0.80211 & 0.59085 & 0.85014 & 0.53504 & 0.86442 \\
\hline $\mathrm{H}$ & 0.76667 & 0.98073 & 0.76201 & 0.96625 & 0.7615 & 0.95153 & $\mathrm{H}$ & 0.70953 & 0.97879 & 0.76153 & 0.98733 & 0.713 & 0.97817 \\
\hline $\mathrm{VH}$ & 0.90982 & 1 & 0.92588 & 1 & 0.92999 & 1 & VH & 0.91148 & 1 & 0.90853 & 1 & 0.90797 & 1 \\
\hline RMSE & 17.4556 & & 17.4346 & & 17.4305 & & RMSE & 17.4570 & & 17.397 & & 17.4479 & \\
\hline Rank & 18 & & 7 & & 3 & & Rank & 21 & & 1 & & 14 & \\
\hline & & 55 & & 10 & II & & & & & & & II & 30 \\
\hline VL & 0 & 0.08531 & 0 & 0.08171 & 0 & 0.09523 & VL & 0 & 0.09022 & 0 & 0.08728 & 0 & 0.09322 \\
\hline L & 0.02848 & 0.29221 & 0.01997 & 0.26076 & 0.03729 & 0.25934 & $\mathrm{~L}$ & 0.02806 & 0.28003 & 0.03211 & 0.25177 & 0.04754 & 0.24385 \\
\hline ML & 0.16226 & 0.48485 & 0.17744 & 0.40947 & 0.16422 & 0.4855 & ML & 0.13755 & 0.49616 & 0.1078 & 0.48675 & 0.13352 & 0.45673 \\
\hline M & 0.33274 & 0.66871 & 0.34997 & 0.68698 & 0.30717 & 0.68502 & M & 0.35659 & 0.67828 & 0.32552 & 0.61039 & 0.3192 & 0.69263 \\
\hline MH & 0.59669 & 0.88884 & 0.52779 & 0.89454 & 0.53659 & 0.81601 & $\mathrm{MH}$ & 0.53507 & 0.87554 & 0.5415 & 0.83595 & 0.544 & 0.81348 \\
\hline $\mathrm{H}$ & 0.70881 & 0.99437 & 0.72383 & 0.96292 & 0.79551 & 0.98406 & $\mathrm{H}$ & 0.71567 & 0.96685 & 0.74822 & 0.96938 & 0.74184 & 0.95353 \\
\hline $\mathrm{VH}$ & 0.91276 & 1 & 0.94355 & 1 & 0.92784 & 1 & $\mathrm{VH}$ & 0.90213 & 1 & 0.92322 & 1 & 0.90431 & 1 \\
\hline RMSE & 17.4711 & & 17.4560 & & 17.4514 & & RMSE & 17.4355 & & 17.4355 & & 17.4361 & \\
\hline Rank & 28 & & 19 & & 17 & & Rank & 8 & & 26 & & 9 & \\
\hline
\end{tabular}




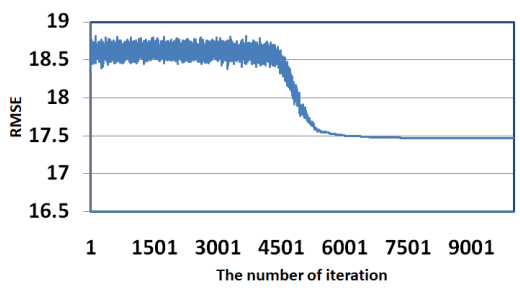

(a)

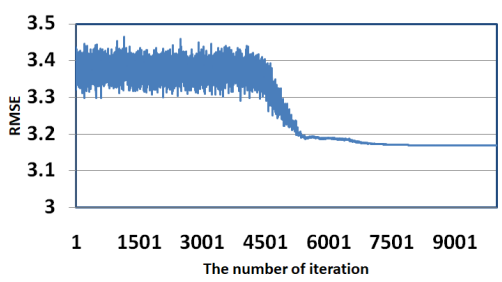

(b)

Figure 6: The progress-plot of mean RMSE, averaged over 30 trials, in time (iteration) for the SA algorithm applied to ID1 using the (a) linguistic weighted sum, and (b) linguistic weighted average approaches.

\subsubsection{Linguistic weighted sum approach}

For the linguistic weighted sum approach, the fuzzy sets obtained for each of the solutions from a trial are provided in Table 12 Considering the RMSE scores, the top three solutions are ID24, ID22, and ID14 yielding the best RMSE scores of $17.3974,17.4267$, and 17.4305 respectively, while ID21 is the worst with an RMSE score of 17.4803. All those solutions indicating the fuzzy sets for the importance of criteria are further illustrated in Figure 7 . We observe almost no similarities between any of the fuzzy sets for the linguistic terms for the top three ranking solutions with the lowest RMSE scores. Moreover, comparing the best and worst solutions ID24 and ID21, respectively, one can notice that there is a slight similarity in the representation for the linguistic term 'Medium', however almost none whatsoever for the other terms. Therefore, based on our observations, evaluating results considering each fuzzy set produced by SA separately is not viable without considering all of the membership functions together and looking into the final ranking of alternatives obtained using the overall approach.

\subsubsection{Linguistic weighted average approach}

For the linguistic weighted average approach, the fuzzy sets for the importance of criteria generated by SA are presented in Table 13 . Considering the RMSE scores, the top three solutions obtained from this approach are ID27, ID25, and ID30 yielding the best RMSE scores of $0.3083,0.3083$, and 0.3085 


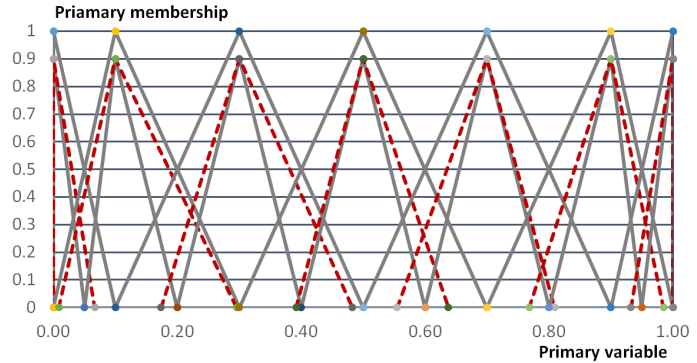

(a)

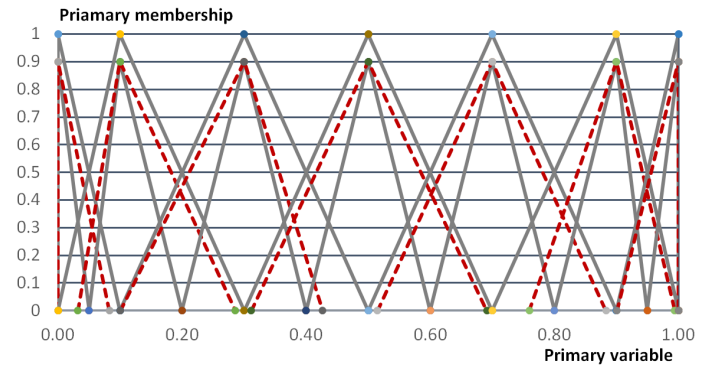

(b)

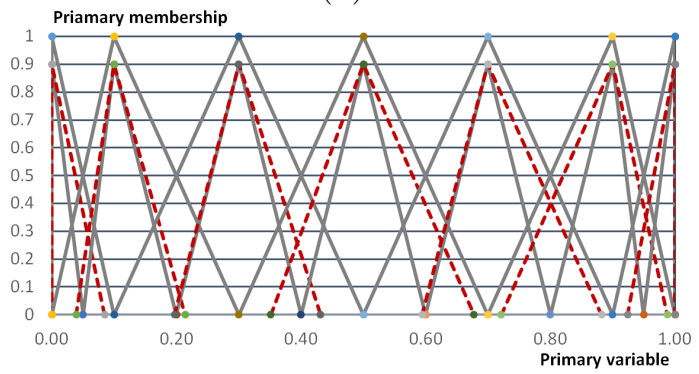

(c)

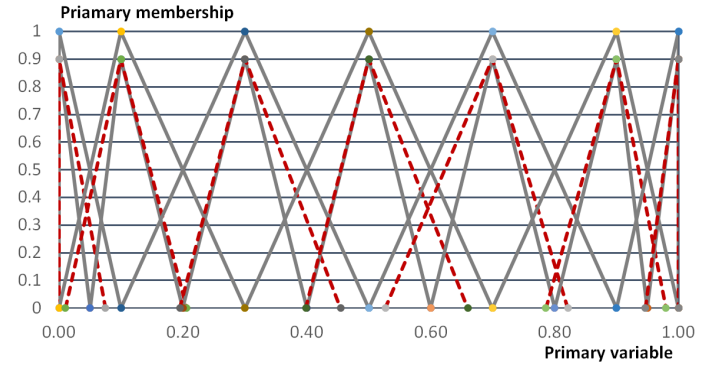

(d)

Figure 7: The top three and worst membership functions for the importance of criteria generated by simulated annealing from various trials are denoted as (a) ID24, (b) ID22, (c) ID14 and (d) ID21, where the linguistic weighted sum approach is used. The lower level of trapezoidal membership function for each linguistic variable is denoted in dashed-line style. 
respectively, while ID18 is the worst with an RMSE score of 0.3091. In Figure 8 (a) ID27, (b) ID25 and (c) ID30, we can observe that the new lower bound of the linguistic term 'Very Low' is narrower when compared to ID18 as illustrated in Figure $8(\mathrm{~d})$. The impact of the differences between membership functions embedded into solutions ID27, ID25, ID30, and ID18 can be seen in Table 13 in terms of RMSE scores. The results show that two different solutions can yield the same RMSE value, e.g., ID27 and ID25. Looking at the generated interval type-2 fuzzy sets, one can observe that the largest differences occur for the linguistic terms 'Medium High' between those two solutions. Thus, once again, there is not much to observe considering each membership function separately as they interact with each other. Hence, we need to consider all membership functions together and look into the final ranking of alternatives obtained using the overall approach.

\subsubsection{Performance comparison of LWS and LWA approaches}

We use the mean ranking scores, averaged over the 30 trials for each alternative garage to comment on the results as illustrated in Table 14 and 15 when LWS and LWA are used, respectively. As expected, SA obtains a solution with different settings for the membership functions and so leading to different ranking scores for garages considering each solution from a trial. One tailed Wilcoxon signed-rank test is applied using the normalised RMSE values from the trials when LWS and LWA are used in SA improved fuzzy approach.

$$
\begin{array}{lc}
H_{0}: & P_{L W S}=P_{L W A} \\
H_{A}: & P_{L W S}<P_{L W A}
\end{array}
$$

where $H_{0}$ represents the null hypothesis which asserts that there is no difference between the medians of the two normalised RMSE distributions. $H_{A}$ represents the alternative hypothesis, i.e, the median normalised RMSE value of the LWS approach is different than the median normalised RMSE value of the LWA approach. The confidence level (significance level) is set to 95\% (p-value under 
Table 13: The widths of fuzzy sets for the importance of criteria for each solution generated by SA when the linguistic weighted average is used. Each solution is reported along with the corresponding RMSE score and rank, where ID\#: solution ID, $a_{1}^{l}$ : Lower bound - first value, $a_{4}^{l}$ : Lower bound - forth value. The top 3 and worst solutions' RMSE scores and ranks are highlighted in bold.

\begin{tabular}{|c|c|c|c|c|c|c|c|c|c|c|c|c|c|}
\hline & $a_{1}^{l}$ & $a_{4}^{l}$ & $a_{1}^{l}$ & $a_{4}^{l}$ & $a_{1}^{l}$ & $a_{4}^{l}$ & & $a_{1}^{l}$ & $a_{4}^{l}$ & $a_{1}^{l}$ & $a_{4}^{l}$ & $a_{1}^{l}$ & $a_{4}^{l}$ \\
\hline LT & \multicolumn{2}{|c|}{ ID1 } & \multicolumn{2}{|c|}{ ID6 } & \multicolumn{2}{|c|}{ ID11 } & LT & \multicolumn{2}{|c|}{ ID16 } & \multicolumn{2}{|c|}{ ID21 } & \multicolumn{2}{|c|}{ ID26 } \\
\hline $\mathrm{VL}$ & 0 & 0.0813 & 0 & 0.0777 & 0 & 0.0527 & VL & 0 & 0.0535 & 0 & 0.0551 & 0 & 0.0976 \\
\hline $\mathrm{L}$ & 0.0292 & 0.2729 & 0.0028 & 0.2180 & 0.0045 & 0.2616 & $\mathrm{~L}$ & 0.0142 & 0.2455 & 0.0146 & 0.2230 & 0.0279 & 0.2972 \\
\hline ML & 0.1113 & 0.4014 & 0.1953 & 0.4939 & 0.1714 & 0.4126 & ML & 0.1825 & 0.4820 & 0.1090 & 0.4391 & 0.1668 & 0.4261 \\
\hline M & 0.3252 & 0.6288 & 0.3721 & 0.6706 & 0.3523 & 0.6299 & M & 0.3195 & 0.6740 & 0.3871 & 0.6402 & 0.3888 & 0.6780 \\
\hline $\mathrm{MH}$ & 0.5192 & 0.8542 & 0.5644 & 0.8717 & 0.5734 & 0.8998 & $\mathrm{MH}$ & 0.5729 & 0.8598 & 0.5843 & 0.8296 & 0.5859 & 0.8469 \\
\hline $\mathrm{H}$ & 0.7087 & 0.9875 & 0.7572 & 0.99789 & 0.7674 & 0.9609 & $\mathrm{H}$ & 0.7155 & 0.9746 & 0.7741 & 0.9841 & 0.7733 & 0.9654 \\
\hline $\mathrm{vH}$ & 0.9206 & 1 & 0.9317 & 1 & 0.9480 & 1 & $\mathrm{VH}$ & 0.9031 & 1 & 0.9028 & 10.9346 & 1 & \\
\hline RMSE & 0.3089 & & 0.3090 & & 0.30869 & & RMSE & 0.3086 & & 0.3088 & & 0.3088 & \\
\hline \multirow[t]{2}{*}{ Rank } & 20 & & 27 & & 7 & & Rank & 6 & & 15 & & 13 & \\
\hline & \multicolumn{2}{|c|}{ ID2 } & \multicolumn{2}{|c|}{ ID7 } & \multicolumn{2}{|c|}{ ID12 } & & \multicolumn{2}{|c|}{ ID17 } & \multicolumn{2}{|c|}{ ID22 } & \multicolumn{2}{|c|}{ ID27 } \\
\hline VL & 0 & 0.0762 & 0 & 0.0848 & 0 & 0.0569 & $\mathrm{VL}$ & 0 & 0.0830 & 0 & 0.0568 & 0 & 0.0613 \\
\hline L & 0.0484 & 0.2798 & 0.0292 & 0.2940 & 0.0019 & 0.2189 & $\mathrm{~L}$ & 0.0364 & 0.2588 & 0.0001 & 0.2024 & 0.0487 & 0.2652 \\
\hline ML & 0.1060 & 0.4905 & 0.1597 & 0.4533 & 0.1647 & 0.4306 & ML & 0.1344 & 0.4570 & 0.1769 & 0.4741 & 0.1646 & 0.4259 \\
\hline M & 0.3606 & 0.6818 & 0.3006 & 0.6538 & 0.3961 & 0.6907 & $\mathrm{M}$ & 0.3532 & 0.6172 & 0.3732 & 0.6319 & 0.3860 & 0.6537 \\
\hline $\mathrm{MH}$ & 0.5981 & 0.8378 & 0.5394 & 0.8764 & 0.5447 & 0.8589 & $\mathrm{MH}$ & 0.5313 & 0.8287 & 0.5407 & 0.8537 & 0.5462 & 0.8685 \\
\hline $\mathrm{H}$ & 0.7778 & 0.9903 & 0.7645 & 0.9590 & 0.7963 & 0.9529 & $\mathrm{H}$ & 0.7096 & 0.9531 & 0.7846 & 0.9899 & 0.7531 & 0.9503 \\
\hline $\mathrm{VH}$ & 0.9142 & 1 & 0.9498 & 1 & 0.9105 & 1 & $\mathrm{VH}$ & 0.9238 & 1 & 0.9131 & 1 & 0.9407 & 1 \\
\hline RMSE & 0.3087 & & 0.3089 & & 0.3088 & & RMSE & 0.3089 & & 0.3089 & & 0.3083 & \\
\hline \multirow[t]{2}{*}{ Rank } & 9 & & 18 & & 14 & & Rank & 23 & & 22 & & 1 & \\
\hline & \multicolumn{2}{|c|}{ ID3 } & \multicolumn{2}{|c|}{ ID8 } & \multicolumn{2}{|c|}{ ID13 } & & & & & 23 & & \\
\hline $\mathrm{VL}$ & 0 & 0.0832 & 0 & 0.0926 & 0 & 0.0509 & $\mathrm{VL}$ & 0 & 0.0808 & 0 & 0.0850 & 0 & 0.0512 \\
\hline $\mathrm{L}$ & 0.0309 & 0.2730 & 0.0342 & 0.2305 & 0.0027 & 0.2186 & $\mathrm{~L}$ & 0.0059 & 0.2034 & 0.0456 & 0.2273 & 0.0116 & 0.2897 \\
\hline ML & 0.1542 & 0.4748 & 0.1234 & 0.4462 & 0.1330 & 0.4950 & ML & 0.1556 & 0.4353 & 0.1980 & 0.4545 & 0.1686 & 0.4964 \\
\hline M & 0.3557 & 0.6983 & 0.3773 & 0.6834 & 0.3296 & 0.6898 & $\mathrm{M}$ & 0.3583 & 0.6254 & 0.3825 & 0.6623 & 0.3602 & 0.6647 \\
\hline $\mathrm{MH}$ & 0.5097 & 0.8680 & 0.5891 & 0.8958 & 0.5225 & 0.8687 & $\mathrm{MH}$ & 0.5065 & 0.8146 & 0.5260 & 0.8696 & 0.5131 & 0.8777 \\
\hline $\mathrm{H}$ & 0.7113 & 0.9708 & 0.7362 & 0.9541 & 0.7772 & 0.9564 & $\mathrm{H}$ & 0.7429 & 0.9928 & 0.7010 & 0.9864 & 0.7827 & 0.9837 \\
\hline $\mathrm{vH}$ & 0.9373 & 1 & 0.9120 & 1 & 0.9187 & 1 & $\mathrm{VH}$ & 0.9151 & 1 & 0.9423 & 1 & 0.9127 & 1 \\
\hline RMSE & 0.3085 & & 0.3089 & & 0.3089 & & RMSE & 0.3091 & & 0.3087 & & 0.3090 & \\
\hline Rank & 4 & & 21 & & 24 & & Rank & 30 & & 12 & & 28 & \\
\hline & & 24 & & D9 & & & & & & & 24 & & \\
\hline $\mathrm{VL}$ & 0 & 0.0904 & 0 & 0.0663 & 0 & 0.0901 & $\mathrm{VL}$ & 0 & 0.0948 & 0 & 0.0979 & 0 & 0.077 \\
\hline $\mathrm{L}$ & 0.0259 & 0.2919 & 0.0031 & 0.2731 & 0.0153 & 0.2246 & $\mathrm{~L}$ & 0.0271 & 0.2792 & 0.0486 & 0.2191 & 0.0454 & 0.2584 \\
\hline ML & 0.1482 & 0.4118 & 0.1754 & 0.4278 & 0.1295 & 0.4084 & ML & 0.1506 & 0.4481 & 0.1783 & 0.4238 & 0.1237 & 0.4160 \\
\hline M & 0.3551 & 0.6974 & 0.3042 & 0.6897 & 0.3614 & 0.6336 & $\mathrm{M}$ & 0.3046 & 0.6772 & 0.3807 & 0.6175 & 0.3610 & 0.6216 \\
\hline $\mathrm{MH}$ & 0.5567 & 0.8507 & 0.5846 & 0.8633 & 0.5394 & 0.8045 & $\mathrm{MH}$ & 0.5519 & 0.8237 & 0.5786 & 0.8999 & 0.5537 & 0.8832 \\
\hline $\mathrm{H}$ & 0.7972 & 0.9778 & 0.7638 & 0.9958 & 0.7043 & 0.9589 & $\mathrm{H}$ & 0.7047 & 0.9569 & 0.7866 & 0.9915 & 0.7833 & 0.9556 \\
\hline $\mathrm{VH}$ & 0.9475 & 1 & 0.9412 & 1 & 0.9217 & 1 & $\mathrm{VH}$ & 0.9397 & 1 & 0.9387 & 1 & 0.9005 & 1 \\
\hline RMSE & 0.3088 & & 0.3087 & & 0.3089 & & RMSE & 0.3086 & & 0.3088 & & 0.3090 & \\
\hline Rank & 16 & & 8 & & 19 & & Rank & 5 & & 17 & & 26 & \\
\hline & & 55 & & 10 & & & & & & & 25 & & \\
\hline $\mathrm{VL}$ & 0 & 0.0781 & 0 & 0.0569 & 0 & 0.0916 & VL & 0 & 0.0946 & 0 & 0.0721 & 0 & 0.0711 \\
\hline $\mathrm{L}$ & 0.0056 & 0.2791 & 0.0181 & 0.2108 & 0.0012 & 0.2934 & $\mathrm{~L}$ & 0.0356 & 0.2736 & 0.0124 & 0.2553 & 0.0136 & 0.2180 \\
\hline ML & 0.1831 & 0.4015 & 0.1304 & 0.4296 & 0.1482 & 0.4227 & $\mathrm{ML}$ & 0.1107 & 0.4851 & 0.1761 & 0.4511 & 0.1918 & 0.4806 \\
\hline M & 0.3304 & 0.6907 & 0.3924 & 0.6627 & 0.3742 & 0.6610 & M & 0.3004 & 0.6792 & 0.3852 & 0.6353 & 0.3218 & 0.6669 \\
\hline MH & 0.5353 & 0.8662 & 0.5578 & 0.8119 & 0.5402 & 0.8449 & MH & 0.5397 & 0.8988 & 0.5705 & 0.8131 & 0.5505 & 0.8659 \\
\hline $\mathrm{H}$ & 0.7545 & 0.9671 & 0.7424 & 0.9675 & 0.7291 & 0.9821 & $\mathrm{H}$ & 0.7503 & 0.9734 & 0.7852 & 0.9652 & 0.7721 & 0.9614 \\
\hline $\mathrm{VH}$ & 0.9468 & 1 & 0.9198 & 1 & 0.9120 & 1 & $\mathrm{VH}$ & 0.9490 & 1 & 0.9037 & 1 & 0.9237 & 1 \\
\hline RMSE & 0.3090 & & 0.3091 & & 0.3087 & & RMSE & 0.3087 & & 0.3083 & & 0.3085 & \\
\hline Rank & 25 & & 29 & & 10 & & Rank & 11 & & 2 & & 3 & \\
\hline
\end{tabular}




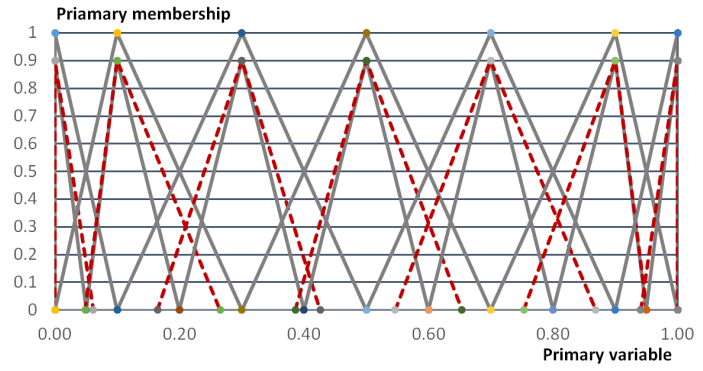

(a)

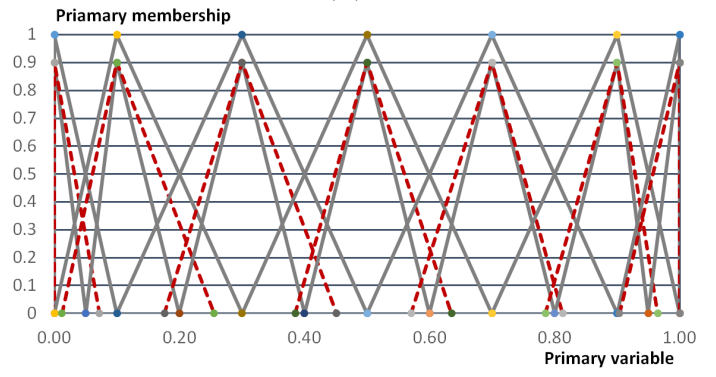

(b)

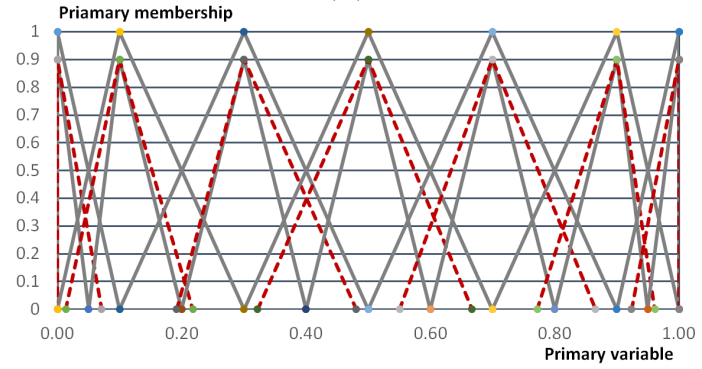

(c)

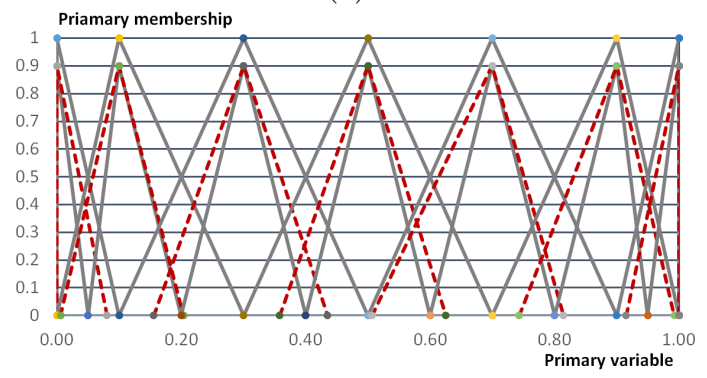

(d)

Figure 8: The top three and worst membership functions for the importance of criteria generated by simulated annealing from various trials are denoted as (a) ID27, (b) ID25, (c) ID30, and (d) ID18 where the linguistic weighted average approach is used. The lower level of trapezoidal membership function for each linguistic variable is denoted in dashed-line style. 
0.05). The Wilcoxon signed-rank test illustrated that there is no statistically significant performance difference between the SA improved fuzzy approaches using LWS and LWA. The overall ranking of garages for each solution is consistently the same with their ranking using the mean scores, regardless of the aggregation method. More importantly, the ranking of garages obtained from the standard interval type-2 fuzzy sets as illustrated in Table 10 is completely different than the ranking of garages obtained from the fuzzy approach improved by SA. type-2 neutrosophic number

The interval type-2 fuzzy sets improved by SA identified that Edirnekapi is the best garage for the electric charging stations and electric buses. Having a mean value of 34.70 for LWS approach and 0.958 for LWA approach, Edirnekapi garage is slightly more appropriate than Ikitelli garage, which has a mean value of 34.22 and 0.824 , respectively. Edirnekapi garage is the closest garage to a BRT station. Edirnekapi BRT station is one of the most crowded BRT stations, especially during peak hours. In terms of economic indicators, Edirnekapi garage has high investment return and revenue potential due to its favorable and close location to dense passenger areas. Its close location to the power grid is another key factor making this location quite appropriate by the experts. Since it already accommodates only the BRT vehicles and has state-of-the-art maintenance and repair facilities inside, accommodating new charging stations and electric vehicles will not necessitate huge transformation costs. Enhanced security infrastructure and expandability add to the capacity of this garage to being able to host electric vehicles. In addition, Table 14 and Table 15 demonstrate that the RMSE scores for all results obtained through improvement by SA regardless of the aggregation method used are better than the result achieved by the interval type- 2 fuzzy sets without using SA as indicated by the 0th row in the tables. The minimum RMSE score is achieved by 24 th solution found as 17.3974 for linguistic weighted sum and 27th solution found as 0.967 for linguistic weighted average, respectively. 
Table 14: Solutions of a linguistic weighted sum approach for ranking potential charging stations using the fuzzy sets obtained by SA. The top three solutions from SA based on RMSE are highlighted and the top three alternatives ranked by the fuzzy approach are shown in bold, italic and underline, respectively from the first to third, for those solutions. The 0th row is obtained from the interval type-2 fuzzy sets directly without applying SA for improvement.

\begin{tabular}{|c|c|c|c|c|c|c|c|}
\hline Solution ID & RMSE & Ikitelli & Edirnekapi & Hasanpasa & Anadolu & Kagithane & Rank \\
\hline 0 & 17.7281 & 39.56 & 36.66 & 24.66 & 32.68 & 28.79 & 31 \\
\hline 1 & 17.4405 & 34.11 & 34.62 & 26.92 & 26.70 & 29.72 & 11 \\
\hline 2 & 17.4758 & 34.61 & 35.06 & 27.26 & 27.06 & 30.14 & 29 \\
\hline 3 & 17.4624 & 34.60 & 35.03 & 27.27 & 27.06 & 30.14 & 25 \\
\hline 4 & 17.4556 & 33.94 & 34.43 & 26.68 & 26.66 & 29.47 & 18 \\
\hline 5 & 17.4711 & 33.96 & 34.43 & 26.71 & 26.67 & 29.50 & 28 \\
\hline 6 & 17.4462 & 34.58 & 35.04 & 27.19 & 27.15 & 30.04 & 13 \\
\hline 7 & 17.4569 & 34.57 & 35.01 & 27.20 & 27.14 & 30.04 & 20 \\
\hline 8 & 17.4582 & 34.75 & 35.21 & 27.39 & 27.21 & 30.25 & 23 \\
\hline 9 & 17.4346 & 34.12 & 34.62 & 26.87 & 26.73 & 29.68 & 7 \\
\hline 10 & 17.4560 & 34.79 & 35.29 & 27.40 & 27.23 & 30.28 & 19 \\
\hline 11 & 17.4571 & 34.77 & 35.25 & 27.40 & 27.22 & 30.26 & 22 \\
\hline 12 & 17.4695 & 34.14 & 34.60 & 26.92 & 26.76 & 29.72 & 27 \\
\hline 13 & 17.4487 & 34.26 & 34.74 & 27.07 & 26.85 & 29.86 & 15 \\
\hline 14 & 17.4305 & 34.12 & 34.61 & 26.89 & 26.74 & $\underline{29.69}$ & 3 \\
\hline 15 & 17.4514 & 34.13 & 34.60 & 26.90 & 26.75 & 29.71 & 17 \\
\hline 16 & 17.4344 & 33.41 & 33.97 & 26.56 & 25.97 & 29.25 & 6 \\
\hline 17 & 17.4620 & 33.44 & 33.98 & 26.58 & 25.99 & 29.27 & 24 \\
\hline 18 & 17.4407 & 33.38 & 33.97 & 26.53 & 25.93 & 29.21 & 12 \\
\hline 19 & 17.4570 & 33.39 & 33.96 & 26.54 & 25.95 & 29.22 & 21 \\
\hline 20 & 17.4355 & 34.38 & 34.84 & 27.04 & 27.02 & 29.86 & 8 \\
\hline 21 & 17.4803 & 34.38 & 34.83 & 27.05 & 27.02 & 29.87 & 30 \\
\hline 22 & 17.4267 & 33.92 & 34.45 & 26.76 & 26.58 & $\underline{29.54}$ & 2 \\
\hline 23 & 17.4510 & 33.94 & 34.45 & 26.78 & 26.60 & 29.56 & 16 \\
\hline 24 & 17.3974 & 34.44 & 34.88 & 27.07 & 27.00 & $\underline{29.93}$ & 1 \\
\hline 25 & 17.4644 & 34.55 & 35.04 & 27.23 & 27.09 & 30.07 & 26 \\
\hline 26 & 17.4314 & 35.09 & 35.49 & 27.58 & 27.48 & 30.50 & 4 \\
\hline 27 & 17.4329 & 34.45 & 34.91 & 27.06 & 27.00 & 29.93 & 5 \\
\hline 28 & 17.4381 & 33.95 & 34.43 & 26.74 & 26.64 & 29.53 & 10 \\
\hline 29 & 17.4479 & 33.97 & 34.44 & 26.77 & 26.65 & 29.55 & 14 \\
\hline 30 & 17.4361 & 34.54 & 35.01 & 27.23 & 27.09 & 30.07 & 9 \\
\hline Mean & 17.4483 & 34.22 & 34.70 & 26.99 & 26.80 & 29.79 & - \\
\hline Std & 0.02 & 0.44 & 0.41 & 0.29 & 0.40 & 0.35 & - \\
\hline
\end{tabular}


Table 15: Solutions of a linguistic weighted average approach for ranking potential charging stations using the fuzzy sets obtained by SA. The top three solutions from SA based on RMSE are highlighted and the top three alternatives ranked by the fuzzy approach with LWA are shown in bold, italic and underline, respectively from the first to third, for those solutions. The 0th row is obtained from the interval type- 2 fuzzy sets directly without applying SA for improvement.

\begin{tabular}{|c|c|c|c|c|c|c|c|}
\hline Solution ID & RMSE & Ikitelli & Edirnekapi & Hasanpasa & Anadolu & Kagithane & Rank \\
\hline 0 & 0.3181 & 0.8890 & 0.8238 & 0.5542 & 0.7344 & 0.6470 & 31 \\
\hline 1 & 0.3089 & 0.8256 & 0.9626 & 0.6140 & 0.4144 & 0.8243 & 20 \\
\hline 2 & 0.3087 & 0.8426 & 0.9529 & 0.6199 & 0.4360 & 0.8414 & 9 \\
\hline 3 & 0.3085 & 0.8373 & 0.9511 & 0.6180 & 0.4137 & 0.8360 & 4 \\
\hline 4 & 0.3088 & 0.8116 & 0.9546 & 0.6434 & 0.4210 & 0.8102 & 16 \\
\hline 5 & 0.3090 & 0.8087 & 0.9525 & 0.6401 & 0.4366 & 0.8073 & 25 \\
\hline 6 & 0.3090 & 0.8214 & 0.9595 & 0.6501 & 0.4265 & 0.8199 & 27 \\
\hline 7 & 0.3089 & 0.8175 & 0.9570 & 0.6467 & 0.4444 & 0.8159 & 18 \\
\hline 8 & 0.3089 & 0.8326 & 0.9619 & 0.6310 & 0.4486 & 0.8314 & 21 \\
\hline 9 & 0.3087 & 0.8260 & 0.9589 & 0.6284 & 0.4238 & 0.8247 & 8 \\
\hline 10 & 0.3091 & 0.8431 & 0.9684 & 0.6368 & 0.4161 & 0.8420 & 29 \\
\hline 11 & 0.3086 & 0.8377 & 0.9651 & 0.6338 & 0.4299 & 0.8365 & 7 \\
\hline 12 & 0.3088 & 0.8143 & 0.9521 & 0.6213 & 0.4334 & 0.8128 & 14 \\
\hline 13 & 0.3089 & 0.8113 & 0.9676 & 0.6189 & 0.4131 & 0.8097 & 24 \\
\hline 14 & 0.3089 & 0.8217 & 0.9565 & 0.6259 & 0.4405 & 0.8203 & 19 \\
\hline 15 & 0.3087 & 0.8178 & 0.9541 & 0.6235 & 0.4181 & 0.8163 & 10 \\
\hline 16 & 0.3086 & 0.8292 & 0.9538 & 0.6136 & 0.4432 & 0.8278 & 6 \\
\hline 17 & 0.3089 & 0.8247 & 0.9519 & 0.6115 & 0.4203 & 0.8233 & 23 \\
\hline 18 & 0.3091 & 0.8393 & 0.9585 & 0.6182 & 0.4136 & 0.8380 & 30 \\
\hline 19 & 0.3086 & 0.8341 & 0.9561 & 0.6158 & 0.4258 & 0.8328 & 5 \\
\hline 20 & 0.3087 & 0.8119 & 0.9608 & 0.6479 & 0.4290 & 0.8105 & 11 \\
\hline 21 & 0.3088 & 0.8091 & 0.9580 & 0.6446 & 0.4475 & 0.8076 & 15 \\
\hline 22 & 0.3089 & 0.8186 & 0.9672 & 0.6147 & 0.4357 & 0.8173 & 22 \\
\hline 23 & 0.3087 & 0.8151 & 0.9640 & 0.6131 & 0.4152 & 0.8137 & 12 \\
\hline 24 & 0.3088 & 0.8297 & 0.9515 & 0.6351 & 0.4171 & 0.8283 & 17 \\
\hline 25 & 0.3083 & 0.8252 & 0.9665 & 0.6320 & 0.4324 & $\underline{0.8237}$ & 2 \\
\hline 26 & 0.3088 & 0.8421 & 0.9556 & 0.6413 & 0.4228 & 0.8408 & 13 \\
\hline 27 & 0.3083 & 0.8346 & 0.9533 & 0.6382 & 0.4397 & $\underline{0.8333}$ & 1 \\
\hline 28 & 0.3090 & 0.8118 & 0.9575 & 0.6244 & 0.4439 & 0.8104 & 28 \\
\hline 29 & 0.3090 & 0.8090 & 0.9551 & 0.6221 & 0.4193 & 0.8075 & 26 \\
\hline 30 & 0.3085 & 0.8210 & 0.9633 & 0.6293 & 0.4121 & $\underline{0.8194}$ & 3 \\
\hline Mean & 0.3088 & 0.8242 & 0.9583 & 0.6284 & 0.4278 & 0.8228 & - \\
\hline Std & 0.0002 & 0.0111 & 0.0053 & 0.0118 & 0.0116 & 0.0112 & - \\
\hline
\end{tabular}


Ikitelli garage is differentiated from the other garages with its substantially larger garage space (see Table 1). This makes it quite suitable for the accommodation of the new electric vehicles and charging infrastructure. However, in terms of economic criteria, Ikitelli garage lags behind the performance of Edirnekapi garage. This raises the problem of economic viability for the investment, even though the capacity characteristics are rather favorable. Ikitelli garage does not perform well in terms of service area characteristics, too. It is not close to bus or BRT stations, nor does it have sufficiently available renewable energy sources. Still, the above-average performance in other energy-related criteria such as the distance from the power grid, reliability of electric power and distance from heavy electricity consumption makes it favorable as a location for the new electric vehicles and charging infrastructure.

Kagithane garage is moderately appropriate (with a mean value of 29.79 and 0.823 for both approaches, respectively) as a potential location. Its poor performance in economic criteria and having inadequate capacity is balanced with a relatively better performance in energy aspects. Kagithane garage has the second largest CNG station for a public bus operator in Europe after Madrid. Its customized design specifically for CNG vehicles makes it less appropriate for accommodating electric vehicles. It is located at the bottom of a valley and has the highest slope connecting it with the main roads, hence makes it considerably unfavorable as a location for electric vehicles.

Hasanpasa and Anadolu garages are the worst options having quite close mean values produced by both of the aggregation approaches. The findings are consistent with their actual characteristics. They are both located in the Asian part of Istanbul, which makes them away from dense passenger areas, having negative implications in terms of economic performance. While Anadolu garage has a relatively larger garage area compared to Hasanpasa garage, it is much far away from bus and BRT lines. Hasanpasa has the smallest garage area amongst all garages, therefore, the least suitable one in terms of capacity considerations. Although it is quite close to one of the terminal BRT stations and already accommodates BRT vehicles, the negative values in other criteria 
offset the gain from this positive aspect.

To summarize, the proposed multi-criteria decision making fuzzy approach improved by SA consistently provides the same garage as the best option for all runs. Considering that SA performing a search based on random moves is a stochastic metaheuristic which could yield a different solution at each run, having this level of consistency with the results increases our confidence in the reliability of our approach. More importantly, the ranking of the alternative garages for placing the battery charging stations resulting from our proposed approach is also confirmed by the IETT experts. Another observation is that there is no performance difference between the two aggregation methods, linguistic weighted sum and average used within the interval type-2 fuzzy sets as they provide the same ranking.

\subsection{Type-1 fuzzy approach versus proposed interval type-2 fuzzy-based ap- proaches}

Interval Type-2 fuzzy sets [4] contains the type-1 fuzzy sets as in [49, investigated in this study. In interval type-2 fuzzy, foot print of uncertainty (FOU) that is somewhat the width of the interval represents the level of uncertainty characterised by the problem. Through the use of SA, we reduce this varying width narrowing the distance between the upper and lower bound of the interval type-2 fuzzy set for each linguistic variable towards a type-1 fuzzy set. SA improves the interval type-2 fuzzy sets based on RMSE and so should be able to return type-1 fuzzy set as a solution being part of the search space, if type-1 fuzzy set produces the best RMSE value. Hence, we compare the type-1 fuzzy approach using the sets provided as in [49] and proposed approaches, first looking into the ranking of alternatives, and then the 'best' RMSE values achieved by them.

Table 16 compares the crisp scores obtained for each alternative using both interval type-2 fuzzy approaches including the one improved by SA based on LWS and LWA aggregation methods and type-1 fuzzy approach [49. The results demonstrate that the top three ranking of alternatives are the same using the 
approach based on type-1 fuzzy sets as well as the proposed approach based on the interval type-2 fuzzy sets improved by SA, as expected.

Table 16: Scores obtained for each alternative using the approaches type-1 fuzzy, interval type-2 fuzzy, and interval type-2 fuzzy improved by SA using different aggregation methods.

\begin{tabular}{lcccccccccc}
\hline & Type-1 fuzzy 49 & \multicolumn{3}{c}{ Interval type-2 fuzzy } & \multicolumn{2}{c}{ Interval type-2 fuzzy improved by SA } \\
\hline Alternatives & Crisp & Rank & LWS & Rank & LWA & Rank & LWS & Rank & LWA & Rank \\
& scores & & scores & & scores & & based SA & & based SA \\
\hline Ikitelli (A1) & 17.39 & 2 & 39.56 & 1 & 0.8890 & 1 & 34.44 & 2 & 0.8346 & 2 \\
Edirnekapi (A2) & 17.51 & 1 & 36.66 & 2 & 0.8238 & 2 & 34.88 & 1 & 0.9533 & 1 \\
Hasanpasa (A3) & 13.87 & 5 & 24.66 & 5 & 0.5542 & 5 & 27.07 & 4 & 0.6382 & 4 \\
Anadolu (A4) & 13.82 & 4 & 32.68 & 3 & 0.7344 & 3 & 27.00 & 5 & 0.4397 & 5 \\
Kagithane (A5) & 15.23 & 3 & 28.79 & 4 & 0.6470 & 4 & 29.93 & 3 & 0.8333 & 3 \\
\hline
\end{tabular}

The maximum score for each of the five alternative is calculated assigning each criterion to Very High using each fuzzy approach. This serves as a reference point for each approach to compute the RMSE value for a given set of scores for the alternatives. The crisp scores are then normalised for each approach yielding the results in Table 17 . The results show that the interval type-2 fuzzy set improved by SA using the LWS aggregation method is obtained the best RMSE value of 0.295 among the all fuzzy approach. The type- 1 fuzzy approach turns out to be the worst one achieving the RMSE value of 0.399. Moreover, because of the sharp decrease in the crisp score of Anadolu (A4), the interval type-2 fuzzy improved by SA along with LWA performs slightly worse than interval type-2 fuzzy using LWA with the RMSE value of 0.296.

Developing a type-1 fuzzy model takes almost the same time as developing the interval type-2 fuzzy model. In this study, we enabled simulated annealing to search for the best interval type-2 fuzzy sets, hence this requires extra time. Each trial using SA has taken about 100 minutes on the machines where the experiments are performed. 
Table 17: Normalised scores and RMSE values for the approaches; type-1 fuzzy, interval type-2 fuzzy, and interval type-2 fuzzy improved by SA using different aggregation methods.

\begin{tabular}{lcccccc}
\hline Approaches & A1 & A2 & A3 & A4 & A5 & RMSE \\
\hline Type-1 fuzzy 49] & 0.104 & 0.101 & 0.211 & 0.213 & 0.165 & $\mathbf{0 . 3 9 9}$ \\
Interval type-2 fuzzy- LWS & 0.034 & 0.060 & 0.242 & 0.107 & 0.165 & $\mathbf{0 . 3 4 9}$ \\
Interval type-2 fuzzy- LWA & 0.012 & 0.031 & 0.199 & 0.071 & 0.125 & $\mathbf{0 . 2 9 6}$ \\
Interval type-2 fuzzy improved by SA - LWS & 0.038 & 0.034 & 0.135 & 0.090 & 0.434 & $\mathbf{0 . 2 9 5}$ \\
Interval type-2 fuzzy improved by SA - LWA & 0.027 & 0.002 & 0.131 & 0.314 & 0.028 & $\mathbf{0 . 3 1 7}$ \\
\hline
\end{tabular}

\section{Conclusion}

The city councils have been increasingly valuing sustainable transportation options considering the societal and environmental benefits and so they have been changing their fleets towards electric vehicles. Hence, the selection of the best location for the deployment of the electric charging stations based on the existing infrastructure has become a key issue to be addressed by the decision-makers in many city councils. This study applies an interval type-2 fuzzy sets improved by simulated annealing (SA) using the linguistic weighted sum and average to solve such a real-world problem instance provided by IETT of Istanbul.

The best configuration for SA is obtained through parameter tuning and it is run for 30 times obtaining 30 different improved membership functions for the importance of criteria. Based on the results, it has been observed that the shape of all membership functions plays a significant role in modeling the problem effectively, and improving them via a search method, such as simulated annealing is possible achieving potentially more reliable results. Based on the mean RMSE score averaging all results from SA over 30 runs show that the proposed approach ranks the options for the garage locations as Edirnekapi, Ikitelli, Kagithane, Hasanpasa, and Anadolu, respectively, from the best to the worst alternative for IETT to install the electric charging stations. The viability and reliability of the proposed approach have been verified by the experts for this particular case study as they have suggested the same ranking 
as the approach. The proposed method provides a feasible approach for tackling this facility location problem and also it is reusable being applicable to other multi-criteria decision making problems. The case study represents a unique application in public sector in the context of a local municipal bus operator, which has been largely overlooked up until now.

There is a growing body of work on the design and development of generalpurpose search methods with reusable components, which has a good crossdomain performance. The goal in this line of research is raising the level of generality of search methods enabling them to be applicable to multiple problem domains with no (the least) modification [50]. The proposed approach is sufficiently general and so reusable. It can be applied to any real world multicriteria decision making problem, ranging from material selection to supplier selection problems.

Hence, based on the same line of thinking as in search methods, the proposed approach can be tested on various MCDM problems to see its cross-domain performance and level of generality. Interestingly, the best-known search algorithm with the best cross-domain performance has been changing. That creates another opportunity, that is replacing simulated annealing with one of the top cross-domain search algorithms is likely to generate an improved performance which is also worth testing as a future study.

\section{References}

[1] Guo S, Zhao H. Optimal site selection of electric vehicle charging station by using fuzzy topsis based on sustainability perspective. Applied Energy 2015;158:390-402.

[2] Alhazmi YA, Mostafa HA, Salama MM. Optimal allocation for electric vehicle charging stations using trip success ratio. International Journal of Electrical Power \& Energy Systems 2017;91:101-16.

[3] Lee C, Han J. Benders-and-price approach for electric vehicle charging 
station location problem under probabilistic travel range. Transportation Research Part B: Methodological 2017;106:130-52.

[4] Adler JD, Mirchandani PB, Xue G, Xia M. The electric vehicle shortestwalk problem with battery exchanges. Networks and Spatial Economics 2016;16(1):155-73.

[5] Liu H, Wang DZ. Locating multiple types of charging facilities for battery electric vehicles. Transportation Research Part B: Methodological 2017;103:30-55.

[6] He X, Zhang S, Ke W, Zheng Y, Zhou B, Liang X, et al. Energy consumption and well-to-wheels air pollutant emissions of battery electric buses under complex operating conditions and implications on fleet electrification. Journal of Cleaner Production 2018;171:714-22.

[7] Zhao H, Li N. Optimal siting of charging stations for electric vehicles based on fuzzy delphi and hybrid multi-criteria decision making approaches from an extended sustainability perspective. Energies 2016;9(4):270.

[8] Abdullah L, Adawiyah C, Kamal C. A decision making method based on interval type-2 fuzzy sets: An approach for ambulance location preference. Applied computing and informatics 2018;14(1):65-72.

[9] Deveci M. Site selection for hydrogen underground storage using interval type-2 hesitant fuzzy sets. International Journal of Hydrogen Energy 2018;43(19):9353-68.

[10] Wu Y, Yang M, Zhang H, Chen K, Wang Y. Optimal site selection of electric vehicle charging stations based on a cloud model and the promethee method. Energies 2016;9(3):157.

[11] Xu J, Zhong L, Yao L, Wu Z. An interval type-2 fuzzy analysis towards electric vehicle charging station allocation from a sustainable perspective. Sustainable cities and society 2018;40:335-51. 
[12] Wu Y, Xie C, Xu C, Li F. A decision framework for electric vehicle charging station site selection for residential communities under an intuitionistic fuzzy environment: A case of beijing. Energies 2017;10(9):1270.

[13] Cui FB, You XY, Shi H, Liu HC. Optimal siting of electric vehicle charging stations using pythagorean fuzzy vikor approach. Mathematical Problems in Engineering 2018;2018:1-12. doi:10.1155/2018/9262067.

[14] Xu J, Zhong L, Yao L, Wu Z. An interval type-2 fuzzy analysis towards electric vehicle charging station allocation from a sustainable perspective. Sustainable Cities and Society 2018;40:335 -51. URL: http: //www.sciencedirect.com/science/article/pii/S2210670717309952 doi https://doi.org/10.1016/j.scs.2017.12.010.

[15] Karasan A, Kaya I, Erdogan M. Location selection of electric vehicles charging stations by using a fuzzy mcdm method: a case study in turkey. Neural Computing and Applications 2018;doi:10.1007/s00521-018-3752-2.

[16] Zadeh L. The concept of a linguistic variable and its applications to approximate reasoning. Inform Science 1975;8:199 - 249.

[17] Oscar C, Patricia M. Intelligent systems with interval type-2 fuzzy logic. International Journal of Innovative Computing, Information and Control 2008;4(4):30 - 48 .

[18] Wu D, Tan WW. Type-2 fls modeling capability analysis. In: The 14th IEEE International Conference on Fuzzy Systems. 2005, p. 242 -7.

[19] Greenfield S, Chiclana F, John R, Coupland S. The sampling method of defuzzification for type-2 fuzzy sets: Experimental evaluation. Information Sciences 2012;189(0):77 - 92 .

[20] Mendel JM, John R, Liu F. Interval type-2 fuzzy logic systems made simple. IEEE T Fuzzy Systems 2006;14(6):808-21. 
[21] Mendel JM. The perceptual computer: the past, up to the present, and into the future. Informatik-Spektrum 2018;41(1):15-26.

[22] D'Alterio P, Garibaldi JM, John R, Pourabdollah A. Constrained interval type-2 fuzzy sets. IEEE Transactions on Fuzzy Systems 2020;:1-.

[23] Garibaldi JM, Guadarrama S. Constrained type-2 fuzzy sets. In: 2011 IEEE Symposium on Advances in Type-2 Fuzzy Logic Systems (T2FUZZ). 2011, p. 66-73.

[24] Zhou H, Ying H, Zhang C. Effects of increasing the footprints of uncertainty on analytical structure of the classes of interval type- 2 mamdani and ts fuzzy controllers. IEEE Transactions on Fuzzy Systems 2019;27(9):188190.

[25] Hassan S, Khosravi A, Jaafar J. The impact of fou size and number of mfs on the prediction performance of interval type-2 fuzzy logic systems. In: 2015 International Symposium on Mathematical Sciences and Computing Research (iSMSC). 2015, p. 104-9.

[26] Kirkpatrick S, Gelatt CD, Vecchi MP. Optimization by simulated annealing. science 1983;220(4598):671-80.

[27] Sörensen K, Glover FW. Metaheuristics. Boston, MA: Springer US; 2013, p. $960-70$.

[28] El-Metwally K, Malik O. Parameter tuning for a fuzzy logic controller. IFAC Proceedings Volumes 1993;26(2, Part 2):581 -4. 12th Triennal Wold Congress of the International Federation of Automatic control. Volume 2 Robust Control, Design and Software, Sydney, Australia, 18-23 July.

[29] Castillo O, Cervantes L, Soria J, Sanchez M, Castro JR. A generalized type-2 fuzzy granular approach with applications to aerospace. Information Sciences 2016;354:165 -77. URL: http://www.sciencedirect. com/science/article/pii/S0020025516301426. doi/https://doi.org/ $10.1016 / j$.ins.2016.03.001. 
[30] Sanchez MA, Castillo O, Castro JR. Information granule formation via the concept of uncertainty-based information with interval type-2 fuzzy sets representation and takagisugenokang consequents optimized with cuckoo search. Applied Soft Computing 2015;27:602 -9. URL: http: //www.sciencedirect.com/science/article/pii/S1568494614002646 doi:https://doi.org/10.1016/j.asoc.2014.05.036.

[31] Cervantes L, Castillo O. Type-2 fuzzy logic aggregation of multiple fuzzy controllers for airplane flight control. Information Sciences 2015;324:247 -56. URL: http://www.sciencedirect. com/science/article/pii/S002002551500479X. doi/https://doi.org/ $10.1016 / j$.ins.2015.06.047

[32] Castillo O, Amador-Angulo L, Castro JR, Garcia-Valdez M. A comparative study of type-1 fuzzy logic systems, interval type-2 fuzzy logic systems and generalized type-2 fuzzy logic systems in control problems. Information Sciences 2016;354:257 -74. URL: http://www. sciencedirect. com/science/article/pii/S002002551630189X. doi/https://doi.org/ $10.1016 / j$. ins.2016.03.026

[33] Sanchez MA, Castillo O, Castro JR. Generalized type-2 fuzzy systems for controlling a mobile robot and a performance comparison with interval type-2 and type-1 fuzzy systems. Expert Systems with Applications 2015;42(14):5904 -14. URL: http://www.sciencedirect. com/science/article/pii/S0957417415002183, doi/https://doi.org/ $10.1016 /$ j.eswa.2015.03.024.

[34] Melin P, Ontiveros E, Gonzlez C, Castro J, Castillo O. An approach for parameterized shadowed type-2 fuzzy membership functions applied in control applications. Soft Computing 2018;:1-15doi 10.1007/ s00500-018-3503-4.

[35] Henderson D, Jacobson SH, Johnson AW. The Theory and Practice of Simulated Annealing. Boston, MA: Springer US; 2003, p. 287-319. 
[36] Jackson WG, Özcan E, John RI. Move acceptance in local search metaheuristics for cross-domain search. Expert Systems with Applications 2018;109:131-51.

[37] IETT - Activity Report. Tech. Rep.; 2016. URL: https://goo.gl/CXEicP

[38] Karnik NN, Mendel JM, Liang Q. Type-2 fuzzy logic systems. Fuzzy Systems, IEEE Transactions on 1999;7(6):643-58.

[39] Gong Y. Fuzzy multi-attribute group decision making method based on interval type-2 fuzzy sets and applications to global supplier selection. International Journal of Fuzzy Systems 2013;15(4).

[40] Mizumoto M, Tanaka K. Fuzzy sets and their operations. Information and Control 1981;48(1):30 - 48. URL: http://www.sciencedirect. com/science/article/pii/S0019995881905787, doi/https://doi.org/ 10.1016/S0019-9958(81)90578-7.

[41] Lian S. Principles of Imprecise-Information Processing: A New Theoretical and Technological System. 1st ed.; Springer Publishing Company, Incorporated; 2016. ISBN 9789811015472.

[42] Wu D, Mendel JM. Aggregation using the linguistic weighted average and interval type-2 fuzzy sets. IEEE Transactions on Fuzzy Systems 2007;15(6):1145-61.

[43] Wu D, Mendel JM. Corrections to aggregation using the linguistic weighted average and interval type-2 fuzzy sets. IEEE Transactions on Fuzzy Systems $2008 ; 16(6): 1664-6$.

[44] Metropolis N, Rosenbluth AW, Rosenbluth MN, Teller AH, Teller E. Equation of state calculations by fast computing machines. Journal of Chemical Physics 1953;21:1087-92.

[45] Kirkpatrick S, Gelatt CD, Vecchi MP. Optimization by simulated annealing. SCIENCE 1983;220(4598):671-80. 
[46] Turk S, Miller S, Özcan E, John R. A simulated annealing approach to supplier selection aware inventory planning. In: IEEE Congress on Evolutionary Computation, CEC 2015, Sendai, Japan, May 25-28, 2015. 2015, p. $1799-806$.

[47] Chen SM, Lee LW. Fuzzy multiple attributes group decision-making based on the ranking values and the arithmetic operations of interval type-2 fuzzy sets. Expert Syst Appl 2010;37(1):824-33.

[48] Taguchi G, Yokoyama Y. Taguchi methods: design of experiments. TAGUCHI METHODS SERIES; ASI Press; 1993. ISBN 9780941243186.

[49] Chen CT. Extensions of the topsis for group decision-making under fuzzy environment. Fuzzy Sets and Systems 2000;114(1):1 - 9 .

[50] Drake JH, Kheiri A, Özcan E, Burke EK. Recent advances in selection hyper-heuristics. European Journal of Operational Research 2020;285(2):405-28. 